\title{
Serendipity in dark photon searches
}

\author{
Philip Ilten, ${ }^{a}$ Yotam Soreq, ${ }^{b}$ Mike Williams $^{c}$ and Wei Xue ${ }^{d}$ \\ ${ }^{a}$ School of Physics and Astronomy, University of Birmingham, \\ Birmingham, B152 2TT, U.K. \\ ${ }^{b}$ Center for Theoretical Physics, Massachusetts Institute of Technology, \\ Cambridge, MA 02139, U.S.A. \\ ${ }^{c}$ Laboratory for Nuclear Science, Massachusetts Institute of Technology, \\ Cambridge, MA 02139, U.S.A. \\ ${ }^{d}$ Theoretical Physics Department, CERN, CH-1211 Geneva 23, Switzerland \\ E-mail: philten@cern.ch, soreqy@mit.edu, mwill@mit.edu, \\ wei.xue@cern.ch
}

ABSTRACT: Searches for dark photons provide serendipitous discovery potential for other types of vector particles. We develop a framework for recasting dark photon searches to obtain constraints on more general theories, which includes a data-driven method for determining hadronic decay rates. We demonstrate our approach by deriving constraints on a vector that couples to the BL current, a leptophobic $B$ boson that couples directly to baryon number and to leptons via $B-\gamma$ kinetic mixing, and on a vector that mediates a protophobic force. Our approach can easily be generalized to any massive gauge boson with vector couplings to the Standard Model fermions, and software to perform any such recasting is provided at https://gitlab.com/philten/darkcast.

Keywords: Phenomenological Models

ARXIV EPRINT: 1801.04847 


\section{Contents}

1 Introduction 1

2 Generic vector boson model 3

$2.1 X$ production 3

$2.2 X$ decays 5

$\begin{array}{lll}2.3 & \text { Efficiency ratios } & 7\end{array}$

3 Example models $\quad 10$

$\begin{array}{ll}3.1 \text { Decays to SM final states } & 10\end{array}$

$\begin{array}{ll}3.2 & \text { Decays to invisible dark-sector final states }\end{array}$

$\begin{array}{llr}4 & \text { Summary } & 15\end{array}$

$\begin{array}{ll}\text { A Additional VMD details } & 16\end{array}$

$\begin{array}{ll}\text { B } X \rightarrow \text { hadrons } & 17\end{array}$

$\begin{array}{ll}\text { C Experiments } & 18\end{array}$

$\begin{array}{lll}\text { C.1 BaBar } & 21\end{array}$

$\begin{array}{lll}\text { C. } 2 & \text { NA48/2 } & 21\end{array}$

$\begin{array}{lll}\text { C.3 Electron bremsstrahlung } & 21\end{array}$

C.4 KLOE 22

$\begin{array}{lll}\text { C.5 } & \mathrm{LHCb} & 22\end{array}$

C.6 Beam dumps 23

$\begin{array}{ll}\text { C.6.1 Electron beam dumps } & 23\end{array}$

C.6.2 Proton beam dumps 24

$\begin{array}{lll}\text { C.7 LEP } & 24\end{array}$

\section{Introduction}

Substantial effort has been dedicated in recent years [1-3] to searching for a massive dark photon, $A^{\prime}$, whose small coupling to the electromagnetic (EM) current arises due to kinetic mixing between the Standard Model (SM) hypercharge and $A^{\prime}$ field strength tensors [4-9]. This mixing provides a potential portal through which dark photons may be produced in the lab, and also via which they can decay into visible SM final states - though decays into invisible dark-sector final states are expected to be dominant if kinematically allowed.

The minimal $A^{\prime}$ model has 3 unknown parameters: the mass of the dark photon, $m_{A^{\prime}}$; the kinetic-mixing strength, $\varepsilon^{2}$; and the dark photon decay branching fraction into invisible dark-sector final states, which is typically assumed to be either 0 or $\approx 1$. Constraints 
have been placed on visible $A^{\prime}$ decays by previous beam-dump [9-23], fixed-target [24-26], collider [27-32], and rare-meson-decay [33-42] experiments, and on invisible $A^{\prime}$ decays in refs. [43-52]. Many ideas have been proposed to further explore the $\left[m_{A^{\prime}}, \varepsilon^{2}\right]$ parameter space in the future [53-67].

Both existing and proposed searches for dark photons provide serendipitous discovery potential for other types of vector particles. Therefore, interpreting these results within the context of a more generic model is well motivated. In this article, we develop a framework for recasting searches for massive vector particles from one model to another, which includes a data-driven method for determining hadronic decay rates. We demonstrate our approach by recasting the existing constraints on dark photons; however, we stress that our approach can easily be applied to any massive gauge boson with vector couplings to the SM fermions.

A variety of production mechanisms have been used in dark-photon searches, which can be categorized as follows:

- bremsstrahlung, eZ $\rightarrow e Z A^{\prime}$ and $p Z \rightarrow p Z A^{\prime}$, using electron and proton beams incident on fixed nuclear targets of charge $Z$;

- annihilation, $e^{+} e^{-} \rightarrow A^{\prime} \gamma$, at $e^{+} e^{-}$colliders;

- Drell-Yan (DY), $q \bar{q} \rightarrow A^{\prime}$, both at hadron colliders and at proton-beam fixed-target experiments;

- meson decays, e.g. $\pi^{0} \rightarrow A^{\prime} \gamma, \eta \rightarrow A^{\prime} \gamma, \omega \rightarrow A^{\prime} \pi^{0}$, and $\phi \rightarrow A^{\prime} \eta$;

- and $V \rightarrow A^{\prime}$ mixing, where $V=\omega, \rho, \phi$ denotes the QCD vector mesons.

Proposed future searches largely exploit the same production mechanisms, though some plan on using positron beams incident on fixed targets for annihilation $[56,68,69]$ or additional meson decays such as $D^{*} \rightarrow D^{0} A^{\prime}$ [62]. Dark photons have been searched for using the following techniques:

- by performing bump hunts in invariant mass spectra using the visible decays $A^{\prime} \rightarrow$ $\ell^{+} \ell^{-}$and $A^{\prime} \rightarrow h^{+} h^{-}$, where thus far $\ell=e, \mu$ and $h=\pi$ have been used;

- by searching for visible displaced $A^{\prime}$ decays, which has been done both at beam dumps and at colliders using secondary vertices;

- and by performing bump hunts in missing mass spectra, which requires the initial state to be known and any visible component of the final state to be detected, providing sensitivity to invisible $A^{\prime}$ decays.

While the production mechanisms and search strategies employed were chosen to achieve the best possible sensitivity to dark photons, each also provides sensitivity to other types of hypothesized vector particles.

The remainder of this article is organized as follows. section 2 develops the framework required to recast these searches, which includes a novel and robust method for determining the hadronic decay rates for $\mathrm{GeV}$-scale bosons. We apply our framework 
to three models in section 3: a vector that couples to the $B-L$ current, a leptophobic $B$ boson that couples directly to baryon number and to leptons via $B-\gamma$ kinetic mixing, and on a vector that mediates a protophobic force. Finally, summary and discussion are provided in section 4. N.b., all information required to recast dark photon searches to any vector model, including software to perform any such recasting, is provided at https://gitlab.com/philten/darkcast.

\section{Generic vector boson model}

In this section, we consider a generic model that couples a vector boson $X$ to SM fermions, $f$, and to invisible dark-sector particles, $\chi$, according to

$$
\mathcal{L} \subset g_{X} \sum_{f} x_{f} \bar{f} \gamma^{\mu} f X_{\mu}+\sum_{\chi} \mathcal{L}_{X \chi \bar{\chi}}
$$

where $g_{X} x_{f}$ is the coupling strength to fermion $f$, and the form of the $X \chi \bar{\chi}$ interaction does not need to be specified. ${ }^{1}$ For example, in the minimal $A^{\prime}$ scenario, where the $A^{\prime}$ coupling to SM fermions arises due to $\gamma-A^{\prime}$ kinetic mixing, $g_{X}=\varepsilon e, x_{\ell}=-1, x_{\nu}=0$, and $x_{q}=2 / 3$ or $-1 / 3$. The $A^{\prime}$ also has a model-dependent coupling to the weak $Z$ current that scales as $\mathcal{O}\left(m_{A^{\prime}}^{2} / m_{Z}^{2}\right)$, see e.g. ref. [70]. For $m_{A^{\prime}}>10 \mathrm{GeV}$, we adopt the model of refs. [71, 72]. The $A^{\prime}$ decays visibly if $m_{A^{\prime}}<2 m_{\chi}$ for all $\chi$, and predominantly invisibly otherwise. The more general model has 14 parameters: the 12 fermion couplings, the $X$ boson mass, $m_{X}$, and its decay branching fraction into invisible dark-sector final states.

Recasting a dark photon search that used the final state $\mathcal{F}$ involves solving the following equation for each $m_{X}=m_{A^{\prime}}$ :

$$
\sigma_{X} \mathcal{B}_{X \rightarrow \mathcal{F}} \epsilon\left(\tau_{X}\right)=\sigma_{A^{\prime}} \mathcal{B}_{A^{\prime} \rightarrow \mathcal{F}} \epsilon\left(\tau_{A^{\prime}}\right),
$$

where $\sigma_{X, A^{\prime}}$ denotes the production cross section, $\mathcal{B}_{X, A^{\prime} \rightarrow \mathcal{F}}$ is the decay branching fraction, and $\epsilon$ is the detector efficiency, whose lifetime dependence is made explicit. From eq. (2.2), one can see that what is needed are the ratios $\sigma_{X} / \sigma_{A^{\prime}}, \mathcal{B}_{X \rightarrow \mathcal{F}} / \mathcal{B}_{A^{\prime} \rightarrow \mathcal{F}}$, and $\epsilon\left(\tau_{X}\right) / \epsilon\left(\tau_{A^{\prime}}\right)$. $N . b$, in models where the $X$ couples to an anomalous SM current, there are additional strong constraints from the $B_{u, d} \rightarrow K X, Z \rightarrow \gamma X$, and $K \rightarrow \pi X$ processes, which arise due to the enhanced production rates of the longitudinal $X$ mode [73-75].

\section{1 $X$ production}

The ratio of production cross sections for both electron-beam bremsstrahlung and $e^{+} e^{-}$ annihilation is

$$
\frac{\sigma_{e Z \rightarrow e Z X}}{\sigma_{e Z \rightarrow e Z A^{\prime}}}=\frac{\sigma_{e^{+} e^{-} \rightarrow X \gamma}}{\sigma_{e^{+} e^{-} \rightarrow A^{\prime} \gamma}}=\frac{\left(g_{X} x_{e}\right)^{2}}{(\varepsilon e)^{2}} .
$$

\footnotetext{
${ }^{1}$ This model is flavor-conserving due to its diagonal couplings. Of course, one could also consider flavor-violating $X$ couplings; however, in such cases, the constraints from studies of flavor-changing neutral currents are much stronger than those from $A^{\prime}$ searches. Furthermore, we only consider real $x_{f}$ for similar reasons, making this a $C P$-conserving model as well.
} 
For proton-beam bremsstrahlung the situation is more complicated, but to a good approximation the ratio can be taken to be

$$
\frac{\sigma_{p Z \rightarrow p Z X}}{\sigma_{p Z \rightarrow p Z A^{\prime}}} \approx \frac{g_{X}^{2}\left(2 x_{u}+x_{d}\right)^{2}}{(\varepsilon e)^{2}}
$$

since only sub-GeV masses have been probed using this production mechanism. The ratio of DY production cross sections involves a sum over quark flavors, $q_{i}$, and is given by

$$
\frac{\sigma_{\mathrm{DY} \rightarrow X}}{\sigma_{\mathrm{DY} \rightarrow A^{\prime}}}=\sum_{q_{i}}\left[\frac{\sigma_{q_{i} \bar{q}_{i} \rightarrow \gamma^{*}}(m)}{\sigma_{\mathrm{DY} \rightarrow \gamma^{*}}(m)}\right]\left[\frac{\sigma_{q_{i} \bar{q}_{i} \rightarrow X}}{\sigma_{q_{i} \bar{q}_{i} \rightarrow A^{\prime}}}\right],
$$

where the first term in the sum is the mass-dependent fraction of the SM DY production attributed to each flavor, and the second term is the contribution from each subprocess

$$
\frac{\sigma_{q_{i} \bar{q}_{i} \rightarrow X}}{\sigma_{q_{i} \bar{q}_{i} \rightarrow A^{\prime}}}=\frac{9\left(g_{X} x_{q_{i}}\right)^{2}}{(\varepsilon e)^{2}} \times \begin{cases}\frac{1}{4} & \text { for } q_{i}=u, c, \\ 1 & \text { for } q_{i}=d, s, b .\end{cases}
$$

For $m_{X} \gtrsim 10 \mathrm{GeV}$, the model-dependent mixing with the $Z$ must be accounted for in eq. (2.6). Furthermore, the value of $e$ should be evaluated at the proper mass scale, though this is a small effect below $m_{Z}$. Determining the fraction of SM DY production attributed to each flavor requires knowledge of the parton distribution functions of the proton, though the uncertainties that arise due to limitations in this knowledge largely cancel in the ratios.

Following ref. [76], we calculate meson-decay ratios using the hidden local symmetries framework of vector meson dominance (VMD) [77], which is successful at predicting lowenergy SM observables. ${ }^{2}$ In this effective theory, external gauge fields - including the SM photon - couple to quarks via mixing with the QCD vector mesons. The ratio of the widths for producing the $X$ and $A^{\prime}$ in decays of the form $V \rightarrow X P$, where $V$ and $P$ denote vector and pseudoscalar mesons, respectively, is given by

$$
\frac{\Gamma_{V \rightarrow X P}}{\Gamma_{V \rightarrow A^{\prime} P}}=\frac{g_{X}^{2}}{(\varepsilon e)^{2}} \frac{\left|\sum_{V^{\prime}} \operatorname{Tr}\left[T_{V} T_{P} T_{V^{\prime}}\right] \operatorname{Tr}\left[T_{V^{\prime}} Q_{X}\right] \mathrm{BW}_{V^{\prime}}\left(m_{X}\right)\right|^{2}}{\left|\sum_{V^{\prime}} \operatorname{Tr}\left[T_{V} T_{P} T_{V^{\prime}}\right] \operatorname{Tr}\left[T_{V^{\prime}} Q\right] \mathrm{BW}_{V^{\prime}}\left(m_{X}\right)\right|^{2}},
$$

where the sum runs over all possible $V P V^{\prime}$ vertices. The quark $\mathrm{U}(3)$-charge matrices are

$$
\begin{aligned}
Q & =\frac{1}{3} \operatorname{diag}\{2,-1,-1\}, \\
Q_{X} & =\operatorname{diag}\left\{x_{u}, x_{d}, x_{s}\right\},
\end{aligned}
$$

and the relevant meson generators, $T_{V, P}$, and the VMD Breit-Wigner form factors, $\mathrm{BW}_{V}(m)$, are detailed in appendix A. When considering $V$ and $P$ from the lowest-lying nonets, where VMD is valid, this reduces to

$$
\frac{\Gamma_{V \rightarrow X P}}{\Gamma_{V \rightarrow A^{\prime} P}}=\left(\frac{g_{X}}{\varepsilon e}\right)^{2} \frac{\left\{\operatorname{Tr}\left[T_{V^{\prime}} Q_{X}\right]\right\}^{2}}{\left\{\operatorname{Tr}\left[T_{V^{\prime}} Q\right]\right\}^{2}}
$$

\footnotetext{
${ }^{2}$ The VMD approach accurately predicts many observables at the 10-20\% level, e.g., the width of the $\omega$ meson [77]. Therefore, we expect that the uncertainty of using VMD and U(3) quark symmetry is $\approx 20 \%$.
} 
where $V^{\prime}$ is chosen such that the process $V \rightarrow V^{\prime} P$ is $\mathrm{SU}(3)$ allowed, e.g. $\omega \rightarrow \omega \eta$ and $\omega \rightarrow \rho \pi^{0}$ are allowed, whereas $\omega \rightarrow \rho \eta$ and $\omega \rightarrow \omega \pi^{0}$ are not. The ratio of widths for $P \rightarrow X \gamma$ and $P \rightarrow A^{\prime} \gamma$ decays satisfies a similar expression:

$$
\frac{\Gamma_{P \rightarrow X \gamma}}{\Gamma_{P \rightarrow A^{\prime} \gamma}}=\left(\frac{g_{X}}{\varepsilon e}\right)^{2} \frac{\left|\sum_{V} \operatorname{Tr}\left[T_{P} Q T_{V}\right] \operatorname{Tr}\left[T_{V} Q_{X}\right] \mathrm{BW}_{V}(m)\right|^{2}}{\left|\sum_{V} \operatorname{Tr}\left[T_{P} Q T_{V}\right] \operatorname{Tr}\left[T_{V} Q\right] \mathrm{BW}_{V}(m)\right|^{2}}
$$

which cannot be reduced into as simple a form due to the fact that multiple terms in the sum over $V$ contribute. Finally, the ratio of production cross sections due to the $X$ mixing with the QCD vector mesons is

$$
\frac{\sigma_{V \rightarrow X}}{\sigma_{V \rightarrow A^{\prime}}}=\frac{g_{X}^{2}}{(\varepsilon e)^{2}} \times \begin{cases}\left(x_{u}-x_{d}\right)^{2} & \text { for } V=\rho, \\ 9\left(x_{u}+x_{d}\right)^{2} & \text { for } V=\omega \\ 9 x_{s}^{2} & \text { for } V=\phi\end{cases}
$$

which is also calculated using VMD. This approach ignores potential interference between the $\rho, \omega$, and $\phi$ production amplitudes. ${ }^{3}$

The sensitivity in many dark photon searches is predominantly due to a single production mechanism at each mass. In such cases, the ratio $\sigma_{X} / \sigma_{A^{\prime}}$ is obtained directly from one of the ratios provided in this subsection. When more than one production mechanism is relevant, the cross-section ratio is

$$
\frac{\sigma_{X}}{\sigma_{A^{\prime}}}=\sum_{i}\left[\frac{\sigma_{A^{\prime}}^{i}}{\sigma_{A^{\prime}}}\right]\left[\frac{\sigma_{X}^{i}}{\sigma_{A^{\prime}}^{i}}\right]
$$

where a Monte Carlo event generator can be used to estimate the relative importance of each production mechanism.

\section{$2.2 \quad X$ decays}

The $X$ boson is assumed to decay predominantly into invisible dark-sector final states if kinematically allowed, and into SM final states otherwise. The partial width of the decay $X \rightarrow f \bar{f}$ is given by

$$
\Gamma_{X \rightarrow f \bar{f}}=\frac{\mathcal{C}_{f}\left(g_{X} x_{f}\right)^{2}}{12 \pi} m_{X}\left(1+\frac{m_{f}^{2}}{m_{X}^{2}}\right) \sqrt{1-4 \frac{m_{f}^{2}}{m_{X}^{2}}},
$$

where $\mathcal{C}_{f}=1$ for $\ell^{+} \ell^{-}, 3$ for $q \bar{q}$, and $1 / 2$ for $\nu \bar{\nu}$; however, for masses $\lesssim 2 \mathrm{GeV}$, we do not expect to obtain a reliable prediction for $\Gamma_{X \rightarrow \text { hadrons }}$ by summing the $q \bar{q}$ contributions from eq. (2.13). Because the $A^{\prime}$ couples to the EM current, its decay rate into hadrons is simply

$$
\Gamma_{A^{\prime} \rightarrow \text { hadrons }}=\Gamma_{A^{\prime} \rightarrow \mu^{+} \mu^{-}} \mathcal{R}_{\mu}\left(m_{A^{\prime}}\right),
$$

\footnotetext{
${ }^{3}$ Including such interference is trivial if the relative phases of the amplitudes are known; however, this production mechanism is only important in hadronic environments, where these phases are generally not known and where interference effects are expected to be negligible.
} 
where $\mathcal{R}_{\mu} \equiv \sigma\left(e^{+} e^{-} \rightarrow\right.$ hadrons $) / \sigma\left(e^{+} e^{-} \rightarrow \mu^{+} \mu^{-}\right)$is known experimentally [78]. N.b., this expression already accounts for $A^{\prime} \rightarrow V$ mixing.

The VMD approach can be used to estimate $\Gamma_{X \rightarrow \mathcal{F}}$ for specific hadronic final states when $m_{X} \lesssim m_{\phi}$, but not in the region from 1 to $2 \mathrm{GeV}$. To obtain reliable predictions for all masses, we have instead developed a data-driven approach based on measured $e^{+} e^{-} \rightarrow \mathcal{F}$ cross sections. First, we normalize each of the most important $e^{+} e^{-} \rightarrow \mathcal{F}$ hadronic cross sections at low mass to that of $e^{+} e^{-} \rightarrow \mu^{+} \mu^{-}$

$$
\mathcal{R}_{\mu}^{\mathcal{F}}(m) \equiv \frac{\sigma_{e^{+} e^{-} \rightarrow \mathcal{F}}}{\sigma_{e^{+} e^{-} \rightarrow \mu^{+} \mu^{-}}}=\frac{9}{\alpha_{\mathrm{EM}}^{2}}\left|\mathcal{A}_{\mathcal{F}}(m)\right|^{2},
$$

where $m$ is the $e^{+} e^{-}$invariant mass. Each $\mathcal{F}$-dependent amplitude is taken to be the sum of a real function $f_{\mathcal{F}}(m)$, which accounts for $V^{*}$ components, and contributions from $V=\rho, \omega, \phi$ as

$$
\mathcal{A}_{\mathcal{F}}(m)=f_{\mathcal{F}}(m) \pm \sum_{V} \mathcal{A}_{\mathcal{F}}^{V}(m)
$$

where the minus sign applies only to $V=\phi$, arising from $\operatorname{Tr}\left[T_{\phi} Q\right]<0$, and the $\mathcal{A}_{\mathcal{F}}^{V}(m)$ amplitudes have Breit-Wigner forms which are provided in appendix B. Taking $f_{\mathcal{F}}(m)$ to be real corresponds to the assumption that the only relevant interference effects between $V^{*}$ and $V$ occur far from the $V^{*}$ poles, which is demonstrated to be a good approximation in appendix B.

The six most important hadronic contributions to $\mathcal{R}_{\mu}$ at low mass are fitted using eq. (2.15), where each $f_{\mathcal{F}}(m)$ is taken to be a bicubic spline with knots every $50 \mathrm{MeV}$ whose values are varied to achieve the best description of the data. Figure 1 shows that these fits describe all data samples well. For $m \lesssim 0.5 \mathrm{GeV}$, the $\pi^{0} \gamma$ final state is the dominant $\omega$ decay mode. Due to a lack of $e^{+} e^{-} \rightarrow \pi^{0} \gamma$ data where this decay is important, we instead calculate this contribution assuming it comes entirely from the $\omega$; i.e. this contribution is estimated as above, but with $f_{\pi^{0} \gamma}(m)=0$ and only using $V=\omega$.

Based on these fits, we are able to decompose $e^{+} e^{-} \rightarrow$ hadrons into $\rho$-like, $\omega$-like, and $\phi$-like contributions, which are discussed in detail in appendix B and shown in figure 2. Each of these contributions is within $20 \%$ of its leading order (LO) perturbative value for $m \gtrsim 1.5 \mathrm{GeV}$, as is $\mathcal{R}_{\mu}$ itself, justifying the use of LO perturbative $\Gamma_{X \rightarrow \text { hadrons }}$ values above $2 \mathrm{GeV}$. Using these $\rho$-like, $\omega$-like, and $\phi$-like models, we can estimate $\Gamma_{X \rightarrow \text { hadrons }}$ for any low-mass $X$ model from

$$
\Gamma_{X \rightarrow \text { hadrons }}=\frac{g_{X}^{2} m_{X}}{12 \pi}\left[\sum_{V} \mathcal{R}_{X}^{V}\left(m_{X}\right)+\mathcal{R}_{X}^{\omega-\phi}\left(m_{X}\right)\right],
$$

where

$$
\begin{aligned}
& \mathcal{R}_{X}^{\rho}(m)=\left\{2 \operatorname{Tr}\left[T_{\rho} Q_{X}\right]\right\}^{2} \mathcal{R}_{\mu}^{\rho}(m), \\
& \mathcal{R}_{X}^{\omega}(m)=\left\{6 \operatorname{Tr}\left[T_{\omega} Q_{X}\right]\right\}^{2} \mathcal{R}_{\mu}^{\omega}(m), \\
& \mathcal{R}_{X}^{\phi}(m)=\left\{3 \sqrt{2} \operatorname{Tr}\left[T_{\phi} Q_{X}\right]\right\}^{2} \mathcal{R}_{\mu}^{\phi}(m) .
\end{aligned}
$$




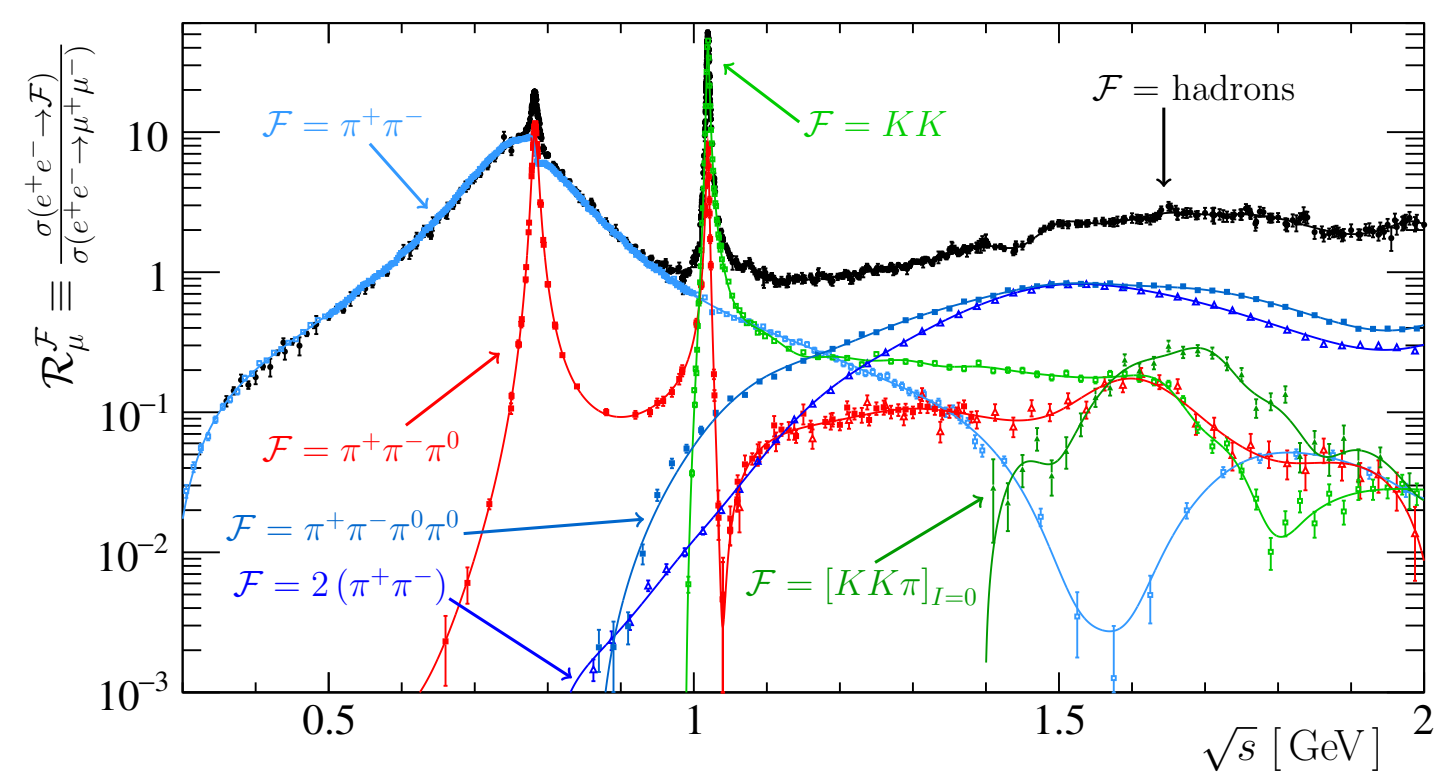

Figure 1. Data used to determine the hadronic decay rates from: the PDG, for the total rate to hadrons [78]; BaBar, for $\pi^{+} \pi^{-}$[79], high-mass $\pi^{+} \pi^{-} \pi^{0}$ [80] (displayed as open triangles), $K K \equiv K^{+} K^{-}+K_{S} K_{L}$ [81], $[K K \pi]_{I=0}$ [82] (i.e. the isoscalar component of the $K K \pi$ final state), $2\left(\pi^{+} \pi^{-}\right)$[83], and $\pi^{+} \pi^{-} \pi^{0} \pi^{0}$ [84]; and from SND, the low-mass $\pi^{+} \pi^{-} \pi^{0}[85,86]$ (displayed as filled squares). See text for discussion on the solid lines.

The final term in eq. (2.17) accounts for interference between the $\omega$-like and $\phi$-like contributions to the $\pi^{+} \pi^{-} \pi^{0}$ final state and is given by

$$
\mathcal{R}_{X}^{\omega-\phi}(m)=36 \sqrt{2} \operatorname{Tr}\left[T_{\omega} Q_{X}\right] \operatorname{Tr}\left[T_{\phi} Q_{X}\right] \Re\left\{\mathcal{A}_{3 \pi}^{\phi}(m)\left[f_{3 \pi}(m)+\mathcal{A}_{3 \pi}^{\omega}(m)\right]^{*}\right\} .
$$

All other interference effects between the $\rho$-like, $\omega$-like, and $\phi$-like contributions are assumed to be negligible. ${ }^{4}$

We reiterate that the approach developed here, specifically eq. (2.17), can be used to obtain $\Gamma_{X \rightarrow \text { hadrons }}$ for any vector model at low mass, where all that is needed as input are the couplings of the $X$ to the $u, d$, and $s$ quarks. Our approach reproduces $\Gamma_{A^{\prime} \rightarrow \text { hadrons }}$ by construction when the model parameters are chosen to be those of the dark photon. While our method invokes a few mild assumptions, this is unavoidable and we believe that the approach developed here is the most robust method for determining the hadronic decay rate of a low-mass vector boson.

\subsection{Efficiency ratios}

The ratio of detector efficiencies for the $X$ relative to the $A^{\prime}$ is taken to be unity for invisible searches. Searches for visible prompt $A^{\prime}$ decays also have the same efficiency for the $X$, provided that $\tau_{X}$ is smaller than the detector decay-time resolution. This is not the case for all models; therefore, lifetime-dependent efficiency effects must be considered even in

\footnotetext{
${ }^{4}$ The numerical values of the $\mathcal{R}_{X}^{\mathcal{F}}$ functions defined in eqs. (2.18)-(2.19) are provided at https://gitlab.com/philten/darkcast.
} 


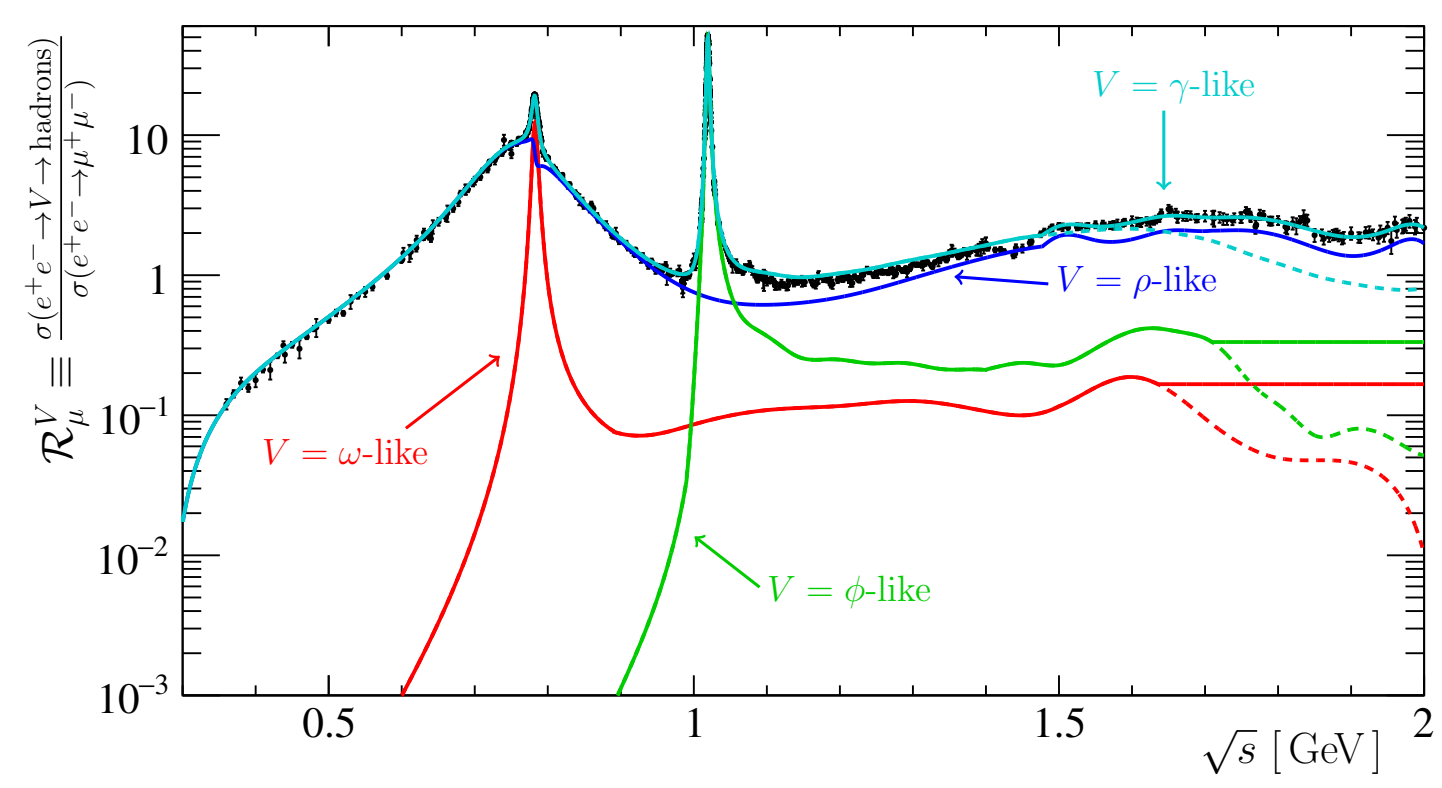

Figure 2. Decomposition of $e^{+} e^{-} \rightarrow$ hadrons, which is of course $\gamma$-like, into $(\rho$-like $)(u \bar{u}-d \bar{d}) / \sqrt{2}$, $(\omega$-like) $(u \bar{u}+d \bar{d}) / \sqrt{2}$, and $(\phi$-like $) s \bar{s}$ contributions. See appendix B for detailed discussion on the derivation of these curves and on the meaning of the dashed lines.

prompt searches. All existing prompt $A^{\prime}$ searches had $\epsilon\left(\tau_{A^{\prime}}\right) \approx 1$ which gives

$$
\frac{\epsilon\left(\tau_{X}\right)}{\epsilon\left(\tau_{A^{\prime}}\right)} \approx 1-e^{-\tilde{t} / \tau_{X}}
$$

where $\tilde{t}$ denotes the largest proper decay time that an $X$ boson could have and still satisfy the prompt $A^{\prime}$ search selection criteria. The experiment-dependent $\tilde{t}$ values are provided in appendix $\mathrm{C}$.

The efficiency ratios are more complicated in searches for long-lived bosons. The recent LHCb search [31] for $A^{\prime} \rightarrow \mu^{+} \mu^{-}$published not only the $A^{\prime}$ exclusion regions, but also the ratio, $r_{\mathrm{ex}}^{\mathrm{ul}}$, of the upper limit on the observed $A^{\prime}$ yield relative to the expected number of observed $A^{\prime}$ decays at each $\left[m_{A^{\prime}}, \varepsilon^{2}\right]$. For the $A^{\prime}$, regions with $r_{\mathrm{ex}}^{\mathrm{ul}}<1$ are excluded. This facilitates recasting the results for each $\tau_{X}=\tau_{A^{\prime}}$, where the ratio of efficiencies is again unity. Regions with

$$
\left[r_{\mathrm{eX}}^{\mathrm{ul}}\left(m_{A^{\prime}}, \varepsilon^{2}\right) \frac{\sigma_{A^{\prime}} \mathcal{B}_{A^{\prime} \rightarrow \mathcal{F}}}{\sigma_{X} \mathcal{B}_{X \rightarrow \mathcal{F}}}\right]_{\tau_{X}=\tau_{A^{\prime}}}<1,
$$

are excluded for the $X$. We encourage future beam dump and displaced-vertex searches to also publish results in this way (or similarly, $r_{\mathrm{ex}}^{\mathrm{ul}}$ at each $\left[m_{A^{\prime}}, \tau_{A^{\prime}}\right]$ ), as it makes recasting the results trivial. N.b., the LHCb sensitivity for some models extends to $\tau_{X}$ values for which $\mathrm{LHCb}$ does not report results, though these regions are easily handled as discussed in appendix C.5.

The published information for constraints placed on dark photons from beam-dump experiments is not sufficient to rigorously recast the results for other models. In principle, the Monte Carlo studies need to be redone, and the $r_{\mathrm{ex}}^{\mathrm{ul}}$ values extracted for each $\left[m_{A^{\prime}}, \varepsilon^{2}\right]$ 
as was done at LHCb [31]. That is beyond the scope of this project. Instead, we set approximate limits by defining an effective proper-time fiducial decay region of $\left[\tilde{t}_{0}, \tilde{t}_{1}\right]$ for each experiment, where $\tilde{t}_{1}$ can be written in terms of the lengths of the decay volume, $L_{\mathrm{dec}}$, and shielding, $L_{\mathrm{sh}}$, as

$$
\tilde{t}_{1}=\tilde{t}_{0}\left(1+L_{\mathrm{dec}} / L_{\mathrm{sh}}\right)
$$

This approach ignores the kinematical spread of the production momentum spectra and the dependence of the efficiency on the location of the decay within the decay volume, though a proper treatment amounts to an $\mathcal{O}(1)$ correction to limits that cover several orders of magnitude for the existing beam-dump results. The probability that a particle with lifetime $\tau$ decays within this fiducial region is given by

$$
\epsilon(\tau)=e^{-\tilde{t}_{0} / \tau}-e^{-\tilde{t}_{1} / \tau}
$$

The values for $\tilde{t}_{0}$ and $\tilde{t}_{1}$ are obtained at each mass from the $A^{\prime}$ limits $\left[\varepsilon_{\min }, \varepsilon_{\max }\right]$ by solving

$$
\varepsilon_{\max }^{2} \epsilon\left[\tau_{A^{\prime}}\left(\varepsilon_{\max }^{2}\right)\right]=\varepsilon_{\min }^{2} \epsilon\left[\tau_{A^{\prime}}\left(\varepsilon_{\min }^{2}\right)\right],
$$

which arises from the fact that the upper limit on observed signal decays is independent of decay time, i.e. the experimental upper limits placed on observed signal decays do not depend on the decay time.

We provide here some simple heuristics that give nearly identical results to the more involved approach described above, provided that the beam-dump experiment is sensitive to the $X$ model being considered at a given mass. For the upper edge of a long-lived $A^{\prime}$ exclusion region, the $A^{\prime}$ lifetime is much smaller than the minimum proper decay time required to enter the beam-dump fiducial region. This means that the efficiency is exponentially suppressed (enhanced) for $\tau_{X}<\tau_{A^{\prime}}\left(\tau_{X}>\tau_{A^{\prime}}\right)$, resulting in the upper edge of the exclusion region for the $X$ occurring at the $g_{X}$ value where

$$
\tau_{X}\left(g_{X}^{\max }\right) \approx \tau_{A^{\prime}}\left(\varepsilon^{\max }\right)
$$

The lower eddge of the $A^{\prime}$ exclusion region is typically where the $A^{\prime}$ lifetime is much larger than the maximum proper decay time required to decay before exiting the fiducial region. In this regime, the ratio of efficiencies is just the ratio of the lifetimes, and the lower edge of the $X$ exclusion region occurs where

$$
\left[\frac{\sigma_{X} \mathcal{B}_{X \rightarrow \mathcal{F}}}{\tau_{X}}\right]_{g_{X}^{\min }} \approx\left[\frac{\sigma_{A^{\prime}} \mathcal{B}_{A^{\prime} \rightarrow \mathcal{F}}}{\tau_{A^{\prime}}}\right]_{\varepsilon^{\min }}
$$

is satisfied. We do not use these heuristics to obtain the results presented in section 3, though they do give nearly identical results except near the high-mass edges of the beamdump exclusion regions, where the large-lifetime approximation is no longer valid at the lower edges of the $A^{\prime}$ exclusion regions. 


\begin{tabular}{|c|cccc|}
\hline Coupling & $A^{\prime}$ & $B-L$ & $B$ & Protophobic \\
\hline$g_{X}$ & $\varepsilon e$ & $g_{B-L}$ & $g_{B}$ & $g_{\nexists}$ \\
$x_{u, c, t}$ & $\frac{2}{3}$ & $\frac{1}{3}$ & $\frac{1}{3}$ & $-\frac{1}{3}$ \\
$x_{d, s, b}$ & $-\frac{1}{3}$ & $\frac{1}{3}$ & $\frac{1}{3}$ & $\frac{2}{3}$ \\
$x_{e, \mu, \tau}$ & -1 & -1 & $-\frac{e^{2}}{(4 \pi)^{2}}$ & -1 \\
$x_{\nu_{e}, \nu_{\mu}, \nu_{\tau}}$ & 0 & -1 & 0 & 0 \\
\hline
\end{tabular}

Table 1. Couplings to SM fermions for the models studied in section 3.

\begin{tabular}{|c|c|c|c|}
\hline Production Mechanism & $B-L$ & $B$ & Protophobic \\
\hline$\frac{\sigma_{e Z} \rightarrow e Z X}{\sigma Z}$ & $\frac{g_{B-L}^{2}}{\overline{s e}^{2}}$ & $\frac{e^{4} g_{B}^{2}}{(4 \pi)^{4}(\rho \rho)^{2}}$ & $\frac{g_{p}^{2}}{(\varepsilon \rho)^{2}}$ \\
\hline $\begin{array}{l}\bar{\sigma}_{e Z \rightarrow e Z A^{\prime}} \\
\sigma_{e^{+} e^{-} \rightarrow X \gamma}\end{array}$ & $\begin{array}{l}(\varepsilon e)^{2} \\
g_{P}^{2}\end{array}$ & $\begin{array}{c}(4 \pi)^{4}(\varepsilon e)^{2} \\
e^{4} q_{D}^{2}\end{array}$ & $\begin{array}{c}(\varepsilon e)^{2} \\
q_{2}^{2}\end{array}$ \\
\hline$\frac{e^{\top} e^{-} \rightarrow X \gamma}{\sigma_{e^{+} e^{-} \rightarrow A^{\prime} \gamma}}$ & $\frac{\frac{3 B-L}{(\varepsilon e)^{2}}}{(1)}$ & $\frac{4 B}{(4 \pi)^{4}(\varepsilon e)^{2}}$ & $\frac{y_{p}}{(\varepsilon e)^{2}}$ \\
\hline$\underline{\sigma_{p Z \rightarrow p Z X}}$ & $g_{B-L}^{2}$ & $g_{B}^{2}$ & 0 \\
\hline$\overline{\sigma_{p Z \rightarrow p Z A^{\prime}}}$ & & $\overline{(\varepsilon e)^{2}}$ & \\
\hline$\sigma_{\{u \bar{u}, c \bar{c}\}} \rightarrow X$ & $g_{B-L}^{2}$ & $g_{B}^{2}$ & $g_{\vec{p}}^{2}$ \\
\hline$\overline{\sigma_{\{u \bar{u}, c \bar{c}\}} \rightarrow A^{\prime}}$ & $4(\varepsilon e)^{2}$ & $\overline{4(\varepsilon e)^{2}}$ & $\overline{4(\varepsilon e)^{2}}$ \\
\hline 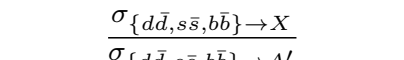 & $\frac{g_{B-L}^{2}}{B-2}$ & $\frac{g_{B}^{2}}{(s \rho)^{2}}$ & $4 g_{p}^{2}$ \\
\hline$\sigma_{\{d \bar{d}, s \bar{s}, b \bar{b}\}} \rightarrow A^{\prime}$ & & & $(\varepsilon e)^{2}$ \\
\hline$\frac{\frac{\sigma_{\rho \rightarrow X}}{\sigma_{\rho \rightarrow A^{\prime}}}}{\sigma_{\rho}}$ & 0 & 0 & $\frac{g_{\vec{p}}^{2}}{(\varepsilon e)^{2}}$ \\
\hline$\frac{\sigma_{\omega \rightarrow X}}{\sigma_{\omega \rightarrow A^{\prime}}}$ & $\frac{4 g_{B-L}^{2}}{(\varepsilon e)^{2}}$ & $\frac{4 g_{B}^{2}}{(\varepsilon e)^{2}}$ & $\frac{g_{p}^{2}}{(\varepsilon e)^{2}}$ \\
\hline$\sigma_{\phi \rightarrow X}$ & $\frac{g_{B-L}^{2}}{B-1}$ & $g_{B}^{2}$ & $4 g_{*}^{2}$ \\
\hline & & $(\varepsilon e)^{2}$ & $(\varepsilon e)^{2}$ \\
\hline
\end{tabular}

Table 2. Production rates for the models in table 1 relative to those of the dark photon, except for meson-decay rates which are provided in table 3 .

\section{Example models}

We now use the framework developed in the previous section to recast existing dark photon searches to obtain constraints on the following models: a vector that couples to the $B-L$ current, a leptophobic $B$ boson that couples directly to baryon number and to leptons via $B-\gamma$ kinetic mixing, and on a vector that mediates a protophobic force [87]. The fermionic couplings of each of these models are provided in table 1. Using these couplings and the results of section 2.1 - including the work in appendix A - it is straightforward to obtain all of the necessary $\sigma_{X} / \sigma_{A^{\prime}}$ ratios, which are summarized in tables 2 and 3. First, we will recast the $A^{\prime}$ searches assuming $\mathcal{B}(X \rightarrow \chi \bar{\chi})=0$ for each of these three models, followed by recasting each of them under the assumption $\mathcal{B}(X \rightarrow \chi \bar{\chi}) \approx 1$. N.b., we do not consider astrophysical constraints in either case (see, e.g., ref. [88]).

\subsection{Decays to SM final states}

For the case where $\mathcal{B}(X \rightarrow \chi \bar{\chi})=0$, it is straightforward to obtain all of the necessary $X$ decay branching fractions to SM final states, which are presented in figure 3 . These 


\begin{tabular}{|c|c|c|}
\hline Decay & $B\left(g_{B} \rightarrow g_{B-L}\right.$ for $\left.B-L\right)$ & Protophobic \\
\hline$\frac{\Gamma_{\rho^{ \pm}, 0 \rightarrow X \pi^{ \pm}, 0}}{\Gamma_{\rho^{ \pm}, 0 \rightarrow A^{\prime} \pi^{ \pm}, 0}}$ & $\frac{4 g_{B}^{2}}{(\varepsilon e)^{2}}$ & $\frac{g_{p}^{2}}{(\varepsilon e)^{2}}$ \\
\hline$\Gamma_{\rho^{0} \rightarrow X \eta}$ & 0 & $g_{p}^{2}$ \\
\hline$\overline{\Gamma_{\rho^{0} \rightarrow A^{\prime} \eta}}$ & & $(\varepsilon e)^{2}$ \\
\hline$\frac{\Gamma_{\omega \rightarrow X \pi^{0}}}{\Gamma_{\omega \rightarrow A^{\prime} \pi^{0}}}$ & 0 & $\frac{g_{\vec{p}}^{2}}{(\varepsilon e)^{2}}$ \\
\hline$\Gamma_{\omega \rightarrow X \eta}$ & $\frac{4 g_{B}^{2}}{\left(\sigma_{0}\right)^{2}}$ & $\frac{g_{p}^{2}}{(-\rho)^{2}}$ \\
\hline$\overline{\Gamma_{\omega \rightarrow A^{\prime} \eta}}$ & $\overline{(\varepsilon e)^{2}}$ & $\overline{(\varepsilon e)^{2}}$ \\
\hline$\frac{\Gamma_{\phi \rightarrow X \eta}}{\Gamma_{\phi \rightarrow A^{\prime} \eta}}$ & $\frac{g_{B}^{2}}{(\varepsilon e)^{2}}$ & $\frac{4 g_{p}^{2}}{(\varepsilon e)^{2}}$ \\
\hline$\Gamma_{\pi^{0} \rightarrow X \gamma}$ & $\left|\mathrm{BW}_{\omega}(m)\right|^{2}$ & $\underline{g_{p}^{2}} \underline{\left|\mathrm{BW}_{\omega}(m)-\mathrm{BW} \rho(m)\right|^{2}} \approx \frac{g_{p}^{2} m^{4}\left(m_{\omega}^{2}-m_{\rho}^{2}\right)^{2}}{2}$ \\
\hline$\overline{\Gamma_{\pi^{0}} \rightarrow A^{\prime} \gamma}$ & $\overline{(\varepsilon e)^{2}} \overline{\left|\mathrm{BW}_{\omega}(m)+\mathrm{BW}_{\rho}(m)\right|^{2}} \sim \overline{(\varepsilon e)^{2}}$ & $\overline{(\varepsilon e)^{2}} \overline{\left|\mathrm{BW}_{\omega}(m)+\mathrm{BW}_{\rho}(m)\right|^{2}} \sim \overline{4(\varepsilon e)^{2}\left(m_{\omega} m_{\rho}\right)^{4}}$ \\
\hline$\frac{\Gamma_{\eta \rightarrow X \gamma}}{\Gamma_{\eta \rightarrow A^{\prime} \gamma}}$ & $\frac{4 g_{B}^{2}}{(\varepsilon e)^{2}} \frac{\left|\mathrm{BW}_{\omega}(m)+\mathrm{BW}_{\phi}(m)\right|^{2}}{\left|\mathrm{BW}_{\omega}(m)+9 \mathrm{BW}_{\rho}(m)-2 \mathrm{BW}_{\phi}(m)\right|^{2}}$ & $\frac{g_{\nexists}^{2}}{(\varepsilon e)^{2}} \mid \frac{\left|\mathrm{BW}_{\omega}(m)-9 \mathrm{BW}_{\rho}(m)+4 \mathrm{BW}_{\phi}(m)\right|^{2}}{\left|\mathrm{BW}_{\omega}(m)+9 \mathrm{BW}_{\rho}(m)-2 \mathrm{BW}_{\phi}(m)\right|^{2}}$ \\
\hline$\Gamma_{\eta^{\prime} \rightarrow X \gamma}$ & $4 g_{B}^{2} \quad\left|\mathrm{BW}_{\omega}(m)-2 \mathrm{BW}_{\phi}(m)\right|^{2}$ & $g_{\nexists p}^{2}\left|\mathrm{BW}_{\omega}(m)-9 \mathrm{BW}_{\rho}(m)-8 \mathrm{BW}_{\phi}(m)\right|^{2}$ \\
\hline$\overline{\Gamma_{\eta^{\prime} \rightarrow A^{\prime} \gamma}}$ & $\overline{(\varepsilon e)^{2}} \frac{\mathrm{BW}_{\omega}(m)+9 \mathrm{BW}_{\rho}(m)+\left.4 \mathrm{BW}_{\phi}(m)\right|^{2}}{2}$ & $\left.e^{2}\right)^{2} \mathrm{BW}_{\omega}(m)+9 \mathrm{BW}_{\rho}(m)+\left.4 \mathrm{BW}_{\phi}(m)\right|^{2}$ \\
\hline
\end{tabular}

Table 3. Meson-decay rates for the models in table 1 relative to those of the dark photon.

are determined using the couplings in table 1 and the results of section 2.2, including the work in appendix B. In addition, we provide the $\mathcal{B}_{B \rightarrow \mathcal{F}}$ values for all important decay modes of the $B$, including specific hadronic final states, in figure 10 of appendix $\mathrm{B}$ as there are plans to use some of these final states in future searches (see, e.g., ref. [89]). The only hadronic final state used in any search considered here is $A^{\prime} \rightarrow \pi^{+} \pi^{-}$, which was employed in the mass region near $m(\omega)$. In this region, we take $\mathcal{B}_{X_{p} \rightarrow \pi^{+} \pi^{-}} \approx \mathcal{B}_{A^{\prime} \rightarrow \pi^{+} \pi^{-}}$, since the $A^{\prime}$ and $X_{p}$ both mix with the $\rho$ with equal strengths. The decays $B-L \rightarrow \pi^{+} \pi^{-}$ and $B \rightarrow \pi^{+} \pi^{-}$require isospin violation, making them difficult to calculate reliably. One expects these branching fractions to be $\mathcal{O}(\%)$; however, we take them to be zero, since the only $A^{\prime} \rightarrow \pi^{+} \pi^{-}$search does not provide competitive sensitivity to $g_{B-L}$ or $g_{B}$.

The searches for visible $A^{\prime}$ decays considered in our study are shown in figure 4 . We do not consider some searches that have inferior sensitivity to others that employed the same production and decay mechanisms. The efficiency ratios are experiment dependent. Detailed discussion on these is provided in appendix C, see also tables 4 and 5 .

The $A^{\prime}$ results recast for $B-L, B$, and the protophobic model are shown in figures $5-7$. Note that for the $B-L$ model, which has nonzero couplings to SM neutrinos, searches for invisible dark photons also provide constraints even for the $\mathcal{B}(B-L \rightarrow \chi \bar{\chi})=0$ case. The recasted $A^{\prime}$ constraints on $B-L$, which are similar to the corresponding $A^{\prime}$ ones, are the strongest on this model in most of the coupling-mass region considered in figure 5. However, we note that recent constraints derived from neutrino experiments, where $B-L$ exchange could compete with the SM neutral-current process, are currently the strongest available in a small region of $g_{B-L}$ values at small masses [92].

For the $B$ model, the constraints bear little resemblance to those on the $A^{\prime}$. The lifetime of the $B$ is much larger than that of the $A^{\prime}$ for $g_{B}=\varepsilon e$ at low masses, due to the fact that the $B$ only couples to leptons via kinetic mixing. One consequence of this is that the LHCb long-lived $A^{\prime}$ search [31] provides much better sensitivity to the $B$ 

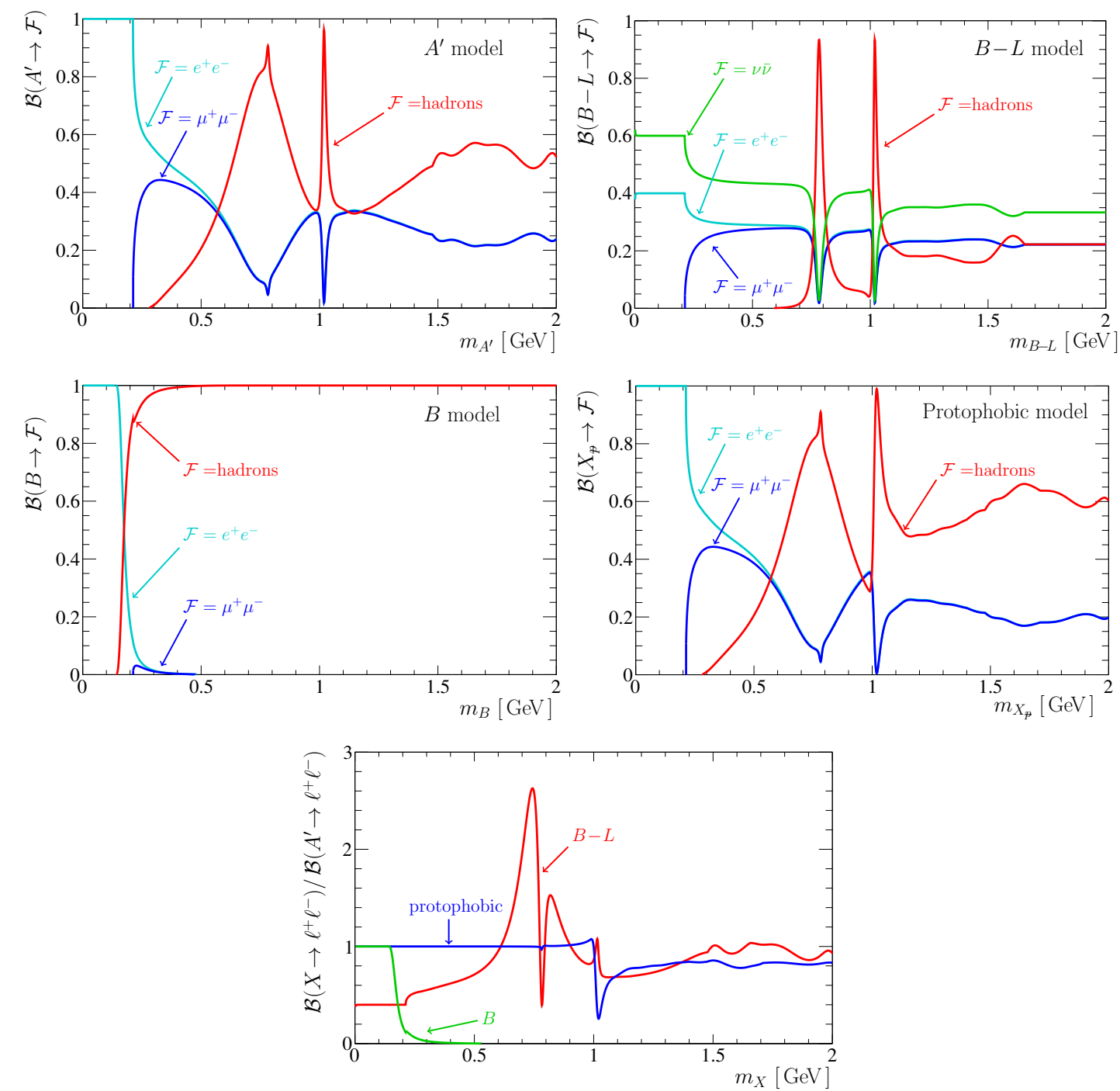

Figure 3. Decay branching fractions for the (top left) $A^{\prime}$, (top right) $B-L$, (middle left) $B$, and (middle right) protophobic models. The branching fractions of the $B$ boson decaying into specific hadronic final states are shown in figure 10. (bottom) Ratio of the branching fractions to leptons for $B-L, B$, and the protophobic model relative to the $A^{\prime}$.

boson than it does to the $A^{\prime}$. Since the $B$ couples to an anomalous SM current, additional strong constraints arise due to the enhanced production rates of the longitudinal $B$ mode as derived in refs. [73, 74]. We have added to these the constraints from the LHCb searches for $B_{u, d} \rightarrow K^{(*)} X$ with $X \rightarrow \mu^{+} \mu^{-}$[93, 94], which provide the strongest non- $A^{\prime}$ limits in the region $2 m_{\mu} \lesssim m_{B} \lesssim 0.6 \mathrm{GeV}$. Additional indirect constraints arise from the requirement of anomaly cancellation by new vector-like fermions, which have not yet been discovered [95]. Under the assumption that the lack of discovery implies that such states do not exist, we apply these constraints following refs. $[73,74]$, which are the strongest non- $A^{\prime}$ constraints in the mass region from about 1 to $5 \mathrm{GeV}$. The recasted $A^{\prime}$ constraints are the strongest on the $B$ at low masses, while the non- $A^{\prime}$-search constraints are dominant for $m_{B} \gtrsim 0.4 \mathrm{GeV}$. 


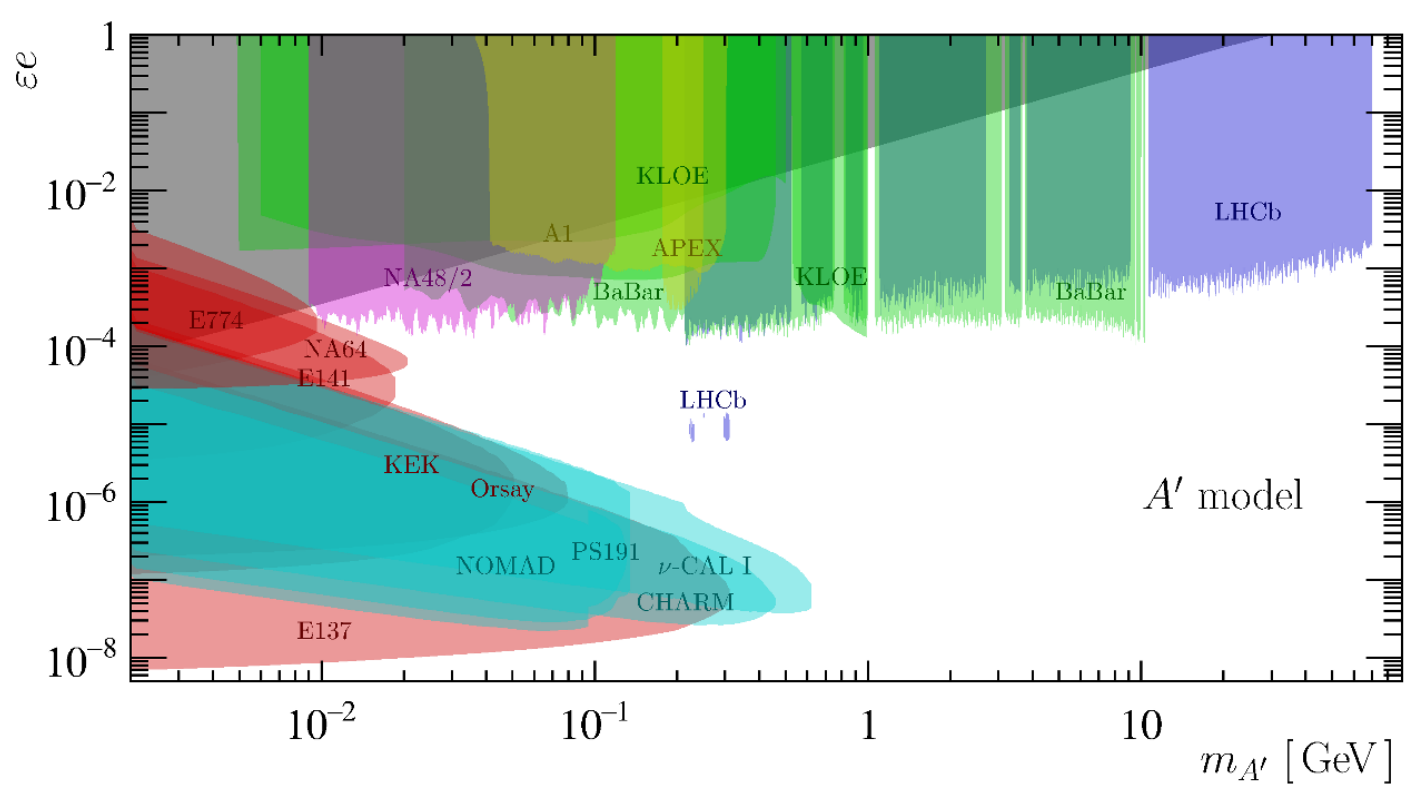

Figure 4. Constraints on visible $A^{\prime}$ decays considered in this study from (red) electron beam dumps, (cyan) proton beam dumps, (green) $e^{+} e^{-}$colliders, (blue) $p p$ collisions, (magenta) meson decays, and (yellow) electron on fixed target experiments. The constraint derived from $(g-2)_{e}$ is shown in grey [90, 91].

The constraints on the protophobic model are similar to those on the $A^{\prime}$, except for the absence of the constraints based on production via proton bremsstrahlung and $\pi^{0}$ decays. The protophobic current is also anomalous in the absence of additional fermions, which means that the constraints from refs. [73, 74] apply to this model as well; however, the coupling to the anomalous current is weaker by a factor of $4 / 9$ due to the different fermionic couplings. ${ }^{5}$ In addition, the sizable differences in the $X_{p}$ lifetime and branching fractions lead to substantial differences in the constraints derived from the anomalous currents. For example, the LHCb $B_{u, d} \rightarrow K^{(*)} X\left(\mu^{+} \mu^{-}\right)$searches [93, 94] provide the strongest constraints in the region $2 m_{\mu} \lesssim m_{X_{\beta}} \lesssim 0.6 \mathrm{GeV}$ for the protophobic model. That said, over most of the coupling-mass region explored thus far, the constraints obtained from $A^{\prime}$ searches are the most stringent.

\subsection{Decays to invisible dark-sector final states}

For the case where $\mathcal{B}(X \rightarrow \chi \bar{\chi}) \approx 1$, only the NA64 [110], BaBar [46], and LEP [52] searches for dark photon decays to invisible final states are used in the recasting. The results are shown in figure 8. Additional constraints on the $B$ model, which couples to an anomalous SM current, arise from $B_{u, d} \rightarrow K X, K \rightarrow \pi X$, and $Z \rightarrow X \gamma$ processes, as studied in refs. $[73,74]$. Recasting these results for the protophobic model, which also couples to an anomalous SM current, simply involves the scale factor of $4 / 9$ discussed in the previous subsection.

\footnotetext{
${ }^{5}$ In the notation of refs. [73, 74], the value of $\mathcal{A}_{X B B}$ is a factor of $4 / 9$ smaller in the protophobic model than in the $B$ model.
} 


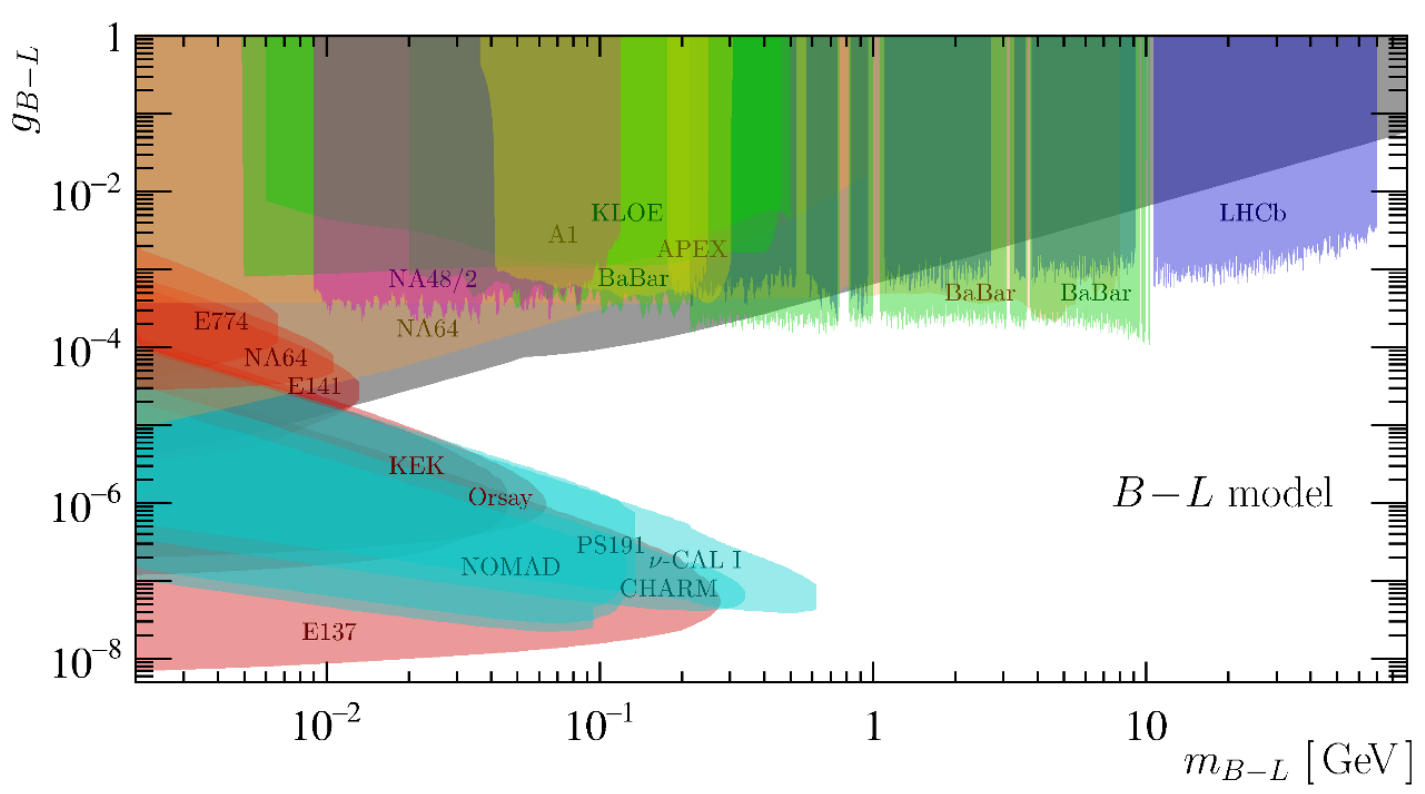

Figure 5. Constraints derived on $B-L$ decays to SM final states using the same experimental color scheme as in figure 4 . The (orange) invisible constraints also apply to $B-L$ due to its coupling to neutrinos. The grey constraints are from Borexino [96, 97], Texono [92, 98], CHARM-II [92, 99], and from SPEAR, DORIS, and PETRA [100, 101].

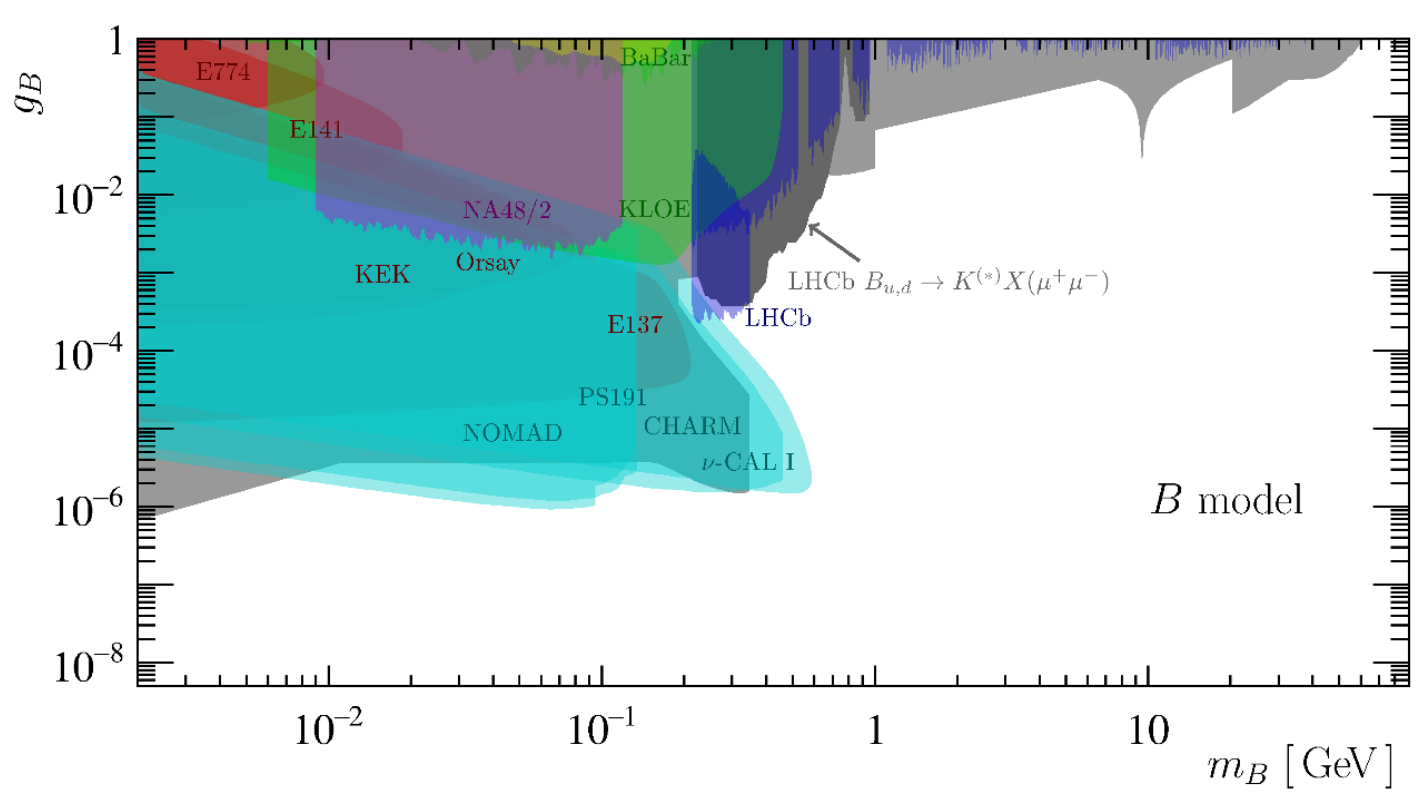

Figure 6. Constraints derived on visible $B$ decays using the same experimental color scheme as in figure 4. The grey constraints come from $\Upsilon[102,103]$ and $\eta[76,104]$ decays, from longitudinalmode enhancements [73, 74] in $B_{u, d} \rightarrow K X$ [105], $K \rightarrow \pi X[106,107]$, and $Z \rightarrow X \gamma[108,109]$ processes, and from the lack of observed new anomaly-canceling fermions [73, 74, 95]. The dark grey constraints, which are obtained in this work following refs. [73, 74], are from the LHCb searches for $B_{u, d} \rightarrow K^{(*)} X$ with $X \rightarrow \mu^{+} \mu^{-}[93,94]$. 


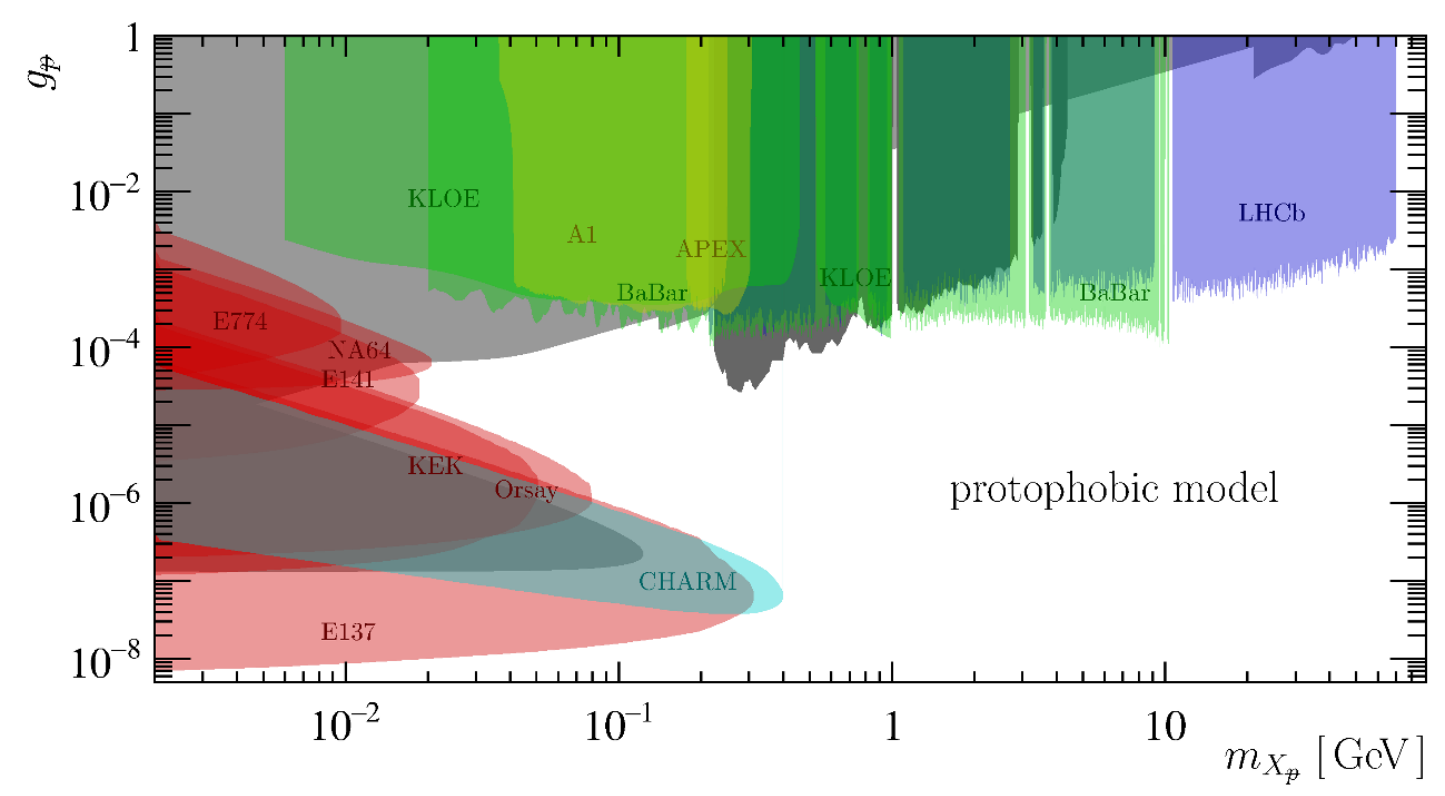

Figure 7. Constraints derived on visible protophobic decays using the same experimental color scheme as in figure 4 . The grey constraints are from the same processes as in figure 6 , but recast to the protophobic model as part of this study.

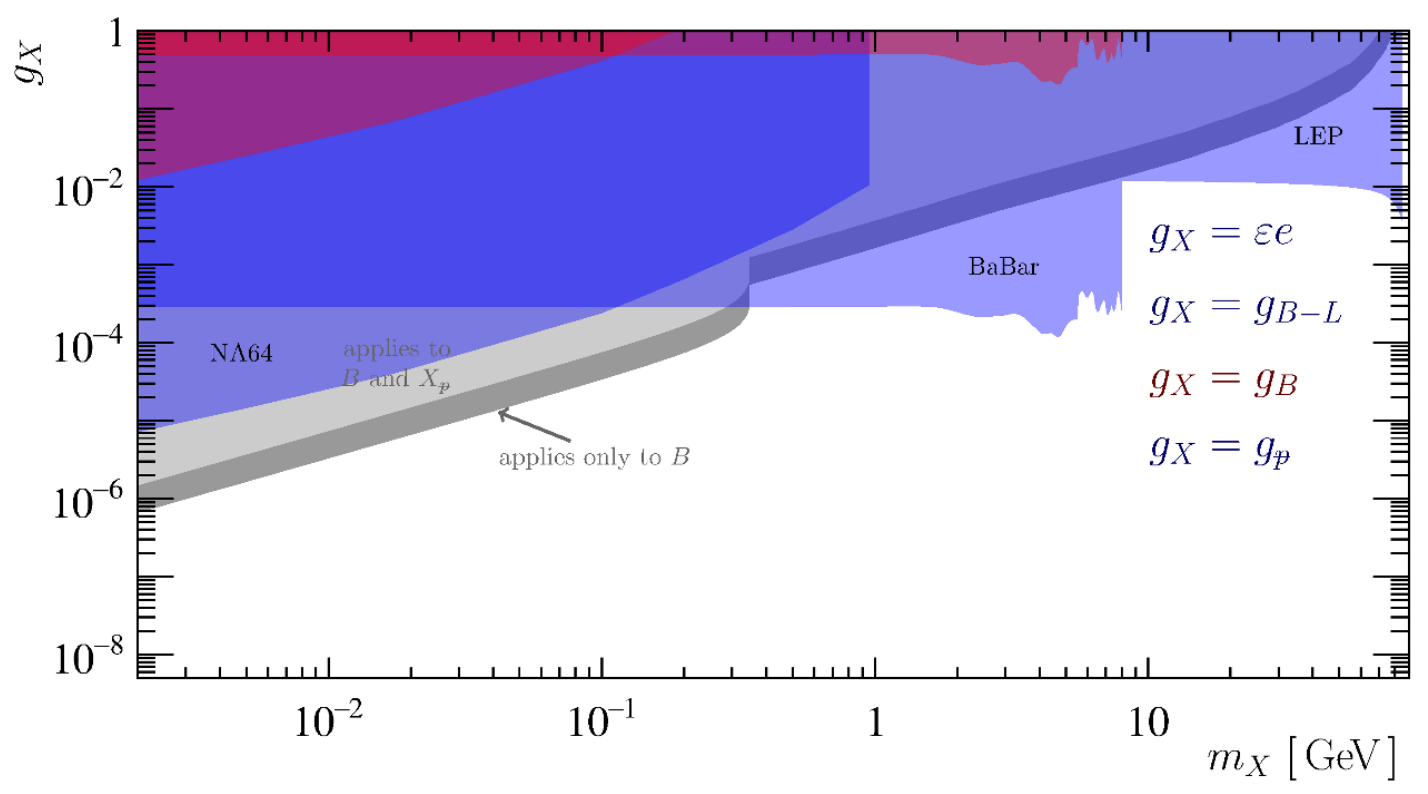

Figure 8. Constraints on all models assuming $\mathcal{B}(X \rightarrow \chi \bar{\chi}) \approx 1$. The grey constraints show the longitudinally enhanced results of refs. $[73,74]$ for $B$, also recast here for the protophobic model.

\section{Summary}

In summary, we have developed a framework for recasting dark photon searches to obtain constraints on more generic models that contain a massive boson with vector couplings to the Standard Model fermions, which includes a data-driven method for determining hadronic decay rates. We demonstrated our approach by deriving constraints on a vector 
that couples to the $B-L$ current, a leptophobic $B$ boson that couples directly to baryon number and to leptons via $B-\gamma$ kinetic mixing, and on a vector that mediates a protophobic force. This framework can easily be generalized to any massive boson with vector couplings to the Standard Model fermions (see, e.g., refs. [111, 112]). Of course, searches for dark photons can also provide sensitivity to non-vector particles $[113,114]$; however, recasting $A^{\prime}$ searches for scalars, etc., does not lend itself to such a simple approach. Finally, all information required to recast dark photon searches to any vector model, including software to perform any such recasting, is provided at https://gitlab.com/philten/darkcast.

\section{Acknowledgments}

We thank Jeff Dror, Stefania Gori, Maxim Pospelov, and Jesse Thaler for providing useful feedback. In addition, we thank Jeff Dror for clarifying how the results in refs. [73, 74] were obtained, Claudia Frugiuele and Elina Fuchs for providing the $B-L$ constraints from ref. [100], Roni Harnik for giving additional information about ref. [52], and Iftah Galon for useful discussion. This work was supported by: PI is supported by a Birmingham Fellowship; YS is supported by the Office of High Energy Physics of the U.S. Department of Energy under grant contract number DE-SC00015476; MW is supported by the U.S. National Science Foundation under contract number PHY-1607225; WX is supported by the grant 669668-NEO-NAT-ERC-AdG-2014.

\section{A Additional VMD details}

In this appendix, we provide additional details about the VMD calculations. The most relevant $\mathrm{U}(3)$ meson generators are

$$
\begin{aligned}
T_{\pi^{0}} & =T_{\rho}=\frac{1}{2} \operatorname{diag}\{1,-1,0\}, \\
T_{\omega} & =\frac{1}{2} \operatorname{diag}\{1,1,0\} \\
T_{\phi} & =\frac{1}{\sqrt{2}} \operatorname{diag}\{0,0,1\} \\
T_{\eta} & \approx \frac{1}{\sqrt{6}} \operatorname{diag}\{1,1,-1\}, \\
T_{\eta^{\prime}} & \approx \frac{1}{2 \sqrt{3}} \operatorname{diag}\{1,1,2\}
\end{aligned}
$$

using $\sin \theta_{\text {mix }}^{\eta, \eta^{\prime}} \approx-1 / 3$ and $\cos \theta_{\text {mix }}^{\eta, \eta^{\prime}} \approx 2 \sqrt{2} / 3$ [115]. The VMD form factors are Breit-Wigner functions taken here to be

$$
\mathrm{BW}_{V}(m)=\frac{m_{V}^{2}}{m_{V}^{2}-m^{2}-i m \Gamma_{V}(m)},
$$

where the mass-dependent widths, which account for changes in the kinematic factors in both the decay amplitudes and phase space collectively denoted by $\mathcal{K}_{\mathcal{F}}(m)$ for the decay 
$V \rightarrow \mathcal{F}$ (see, e.g., refs. [81, 85, 86] for these kinematic factors), are

$$
\Gamma_{V}(m)=\sum_{\mathcal{F}} \mathcal{B}_{V \rightarrow \mathcal{F}} \Gamma_{V}\left(m_{V}\right) \frac{\mathcal{K}_{\mathcal{F}}(m)}{\mathcal{K}_{\mathcal{F}}\left(m_{V}\right)}
$$

The following final states are considered for $\Gamma_{V}(m): \pi^{+} \pi^{-}$for the $\rho ; \pi^{+} \pi^{-} \pi^{0}, \pi^{0} \gamma$, and $\pi^{+} \pi^{-}$for the $\omega$; and $K^{+} K^{-}, K_{S} K_{L}, \pi^{+} \pi^{-} \pi^{0}$, and $\eta \gamma$ for the $\phi$. Finally, for both gauged $B-L$ and $B$, the quark couplings are universal and given by

$$
Q_{B-L}=Q_{B}=\frac{1}{3} \operatorname{diag}\{1,1,1\}
$$

while for the protophobic force the quark-coupling matrix is

$$
Q_{p}=\frac{1}{3} \operatorname{diag}\{-1,2,2\} .
$$

The most relevant decay rates for producing these bosons are listed in table 3 .

\section{B $\quad X \rightarrow$ hadrons}

To obtain reliable predictions of $\Gamma_{X \rightarrow \text { hadrons }}$ for low masses, we have developed a datadriven approach based on measured $e^{+} e^{-} \rightarrow \mathcal{F}$ cross sections. As stated above, we first normalize each of the most important low-mass hadronic $e^{+} e^{-} \rightarrow \mathcal{F}$ cross sections to that of $e^{+} e^{-} \rightarrow \mu^{+} \mu^{-}$according to eq. (2.15). The $\mathcal{A}_{\mathcal{F}}^{V}$ amplitudes in eq. (2.16) are given by

$$
\mathcal{A}_{\mathcal{F}}^{V}(m)=\frac{\Gamma_{V}}{m_{V}} \mathrm{BW}_{V}(m) \sqrt{\frac{\mathcal{B}_{V \rightarrow e^{+} e^{-}} \mathcal{B}_{V \rightarrow \mathcal{F}} \mathcal{K}_{\mathcal{F}}(m)}{\mathcal{K}_{\mathcal{F}}\left(m_{V}\right)}} .
$$

We then fit the $e^{+} e^{-} \rightarrow \mathcal{F}$ cross-section data for the most important hadronic final states, and use these results to decompose $e^{+} e^{-} \rightarrow$ hadrons into $\rho$-like, $\omega$-like, and $\phi$-like contributions (see figure 2) defined as:

- The dashed $\gamma$-like line shows the sum of all final states considered here, including $\pi^{0} \gamma$, which overshoots (undershoots) the PDG $\mathcal{R}_{\mu}$ data for $m \lesssim 1.5 \mathrm{GeV}(m \gtrsim 1.5 \mathrm{GeV})$. The PDG result was produced in 2003, and it does not include any of the highprecision data used in our study. ${ }^{6}$ We take the total $e^{+} e^{-} \rightarrow$ hadrons - the solid $\gamma$ like line in figure 2- to be our sum below $1.48 \mathrm{GeV}$ and the PDG version otherwise, since at higher masses decay modes not included in our study are expected to be important.

- The dashed $\omega$-like curve includes the $\omega \rightarrow \pi^{0} \gamma$ contribution, along with the model used to fit the $\pi^{+} \pi^{-} \pi^{0}$ data but with the $\phi$ amplitude removed. Interference between the $\phi$ and $f_{\pi^{+} \pi^{-} \pi^{0}}(m)$ terms causes the large visible dip near $1.05 \mathrm{GeV}$, which is far

\footnotetext{
${ }^{6}$ Ref. [84] shows a comparison of the recent BaBar $\pi^{+} \pi^{-} \pi^{0} \pi^{0}$ data to the older data used to make the PDG average, where one can see that the dip in the PDG data at $m \approx 1.45 \mathrm{GeV}$ is most likely an experimental artifact that arose due to a confluence of experimental thresholds.
} 
from any $\omega^{*}$ poles justifying the use of a real $f_{\pi^{+} \pi^{-} \pi^{0}}(m)$ function. The LO perturbative value of $\mathcal{R}_{\mu}^{\omega}$ is $1 / 6$. The $\omega$-like curve overshoots this slightly near $1.6 \mathrm{GeV}$, which is not unexpected given that there are several $\omega^{*}$ poles nearby, then falls rapidly at higher masses. We assume that this fall off is due to additional (neglected) final states becoming important, and augment the $\omega$-like contribution (solid curve) to take on the LO perturbative value for $m \gtrsim 1.6 \mathrm{GeV}$.

- The dashed $\phi$-like curve includes the $K K$ and $[K K \pi]_{I=0}$ contributions, along with $\phi \rightarrow \pi^{+} \pi^{-} \pi^{0}$. The LO perturbative value of $\mathcal{R}_{\mu}^{\phi}$ is $1 / 3$. Similarly to the $\omega$-like curve, the $\phi$-like curve is expected to overshoot the LO perturbative value near the $\phi(1680)$, and the fact that it falls off at higher masses is assumed to be due to neglected final states. We augment the $\phi$-like contribution (solid curve) to take on the LO perturbative value for $m \gtrsim 1.7 \mathrm{GeV}$.

- Finally, the $\rho$-like contribution is assumed to be entirely described by the $\pi^{+} \pi^{-}$and $4 \pi$ data for $m<1.1 \mathrm{GeV}$, and is defined as the (solid) $\gamma$-like contribution with the (solid) $\omega$-like and $\phi$-like curves subtracted for $m>1.1 \mathrm{GeV}$. The resulting $\rho$-like curve is within $10 \%$ of its $\mathrm{LO}$ perturbative value of $3 / 2$ for $m \gtrsim 1.8 \mathrm{GeV} .^{7}$

We can further justify the use of the LO perturbative values at higher masses by the fact that $\mathcal{R}_{\mu}$ itself is within $20 \%$ of its LO perturbative value of 2 for $m \gtrsim 1.5 \mathrm{GeV}$.

Using these $\rho$-like, $\omega$-like, and $\phi$-like models, we can estimate $\Gamma_{X \rightarrow \text { hadrons }}$ for any $X$ model using eq. (2.17). Figure 9 shows $\Gamma_{X \rightarrow \text { hadrons }}$ for a dark photon, along with for the $B-L, B$, and protophobic models. By construction, our approach gives the canonical $\Gamma_{A^{\prime} \rightarrow \text { hadrons }}$ result for the dark photon model. ${ }^{8}$ Since $B-L$ and $B$ do not mix with the $\rho$, their hadronic decay rates are substantially lower, especially at lower masses. Note that the $\phi-\omega$ interference dip is below the $\phi$ peak for these models, since the relative sign between the $\omega$ and $\phi$ amplitudes is positive here versus negative for the $A^{\prime}$ model. The protophobic model has a similar hadronic decay width to the $A^{\prime}$ below the $\phi$; however, at larger masses its width is larger due to its larger $s$-quark coupling. Finally, we also provide the $\mathcal{B}_{B \rightarrow \mathcal{F}}$ values for all important decay modes of the $B$, including specific hadronic final states, in figure 10 as there are plans to use some of these final states in future searches [89].

\section{Experiments}

This section contains all of the experiment-specific information, which is summarized tables 4 and 5 .

\footnotetext{
${ }^{7}$ This approach attributes all of the $\rho-\omega$ mixing in the $\pi^{+} \pi^{-}$final state to the $\rho$-like current. While one could certainly question the validity of this choice, the level at which isospin violation occurs in vector mesons is small compared to the overall precision of the VMD calculations for production rates; therefore, it is acceptable to neglect this complication when recasting the dark photon results.

${ }^{8} \mathrm{With}$ the caveat of using an updated $\mathcal{R}_{\mu}$ for $m \lesssim 1.6 \mathrm{GeV}$.
} 


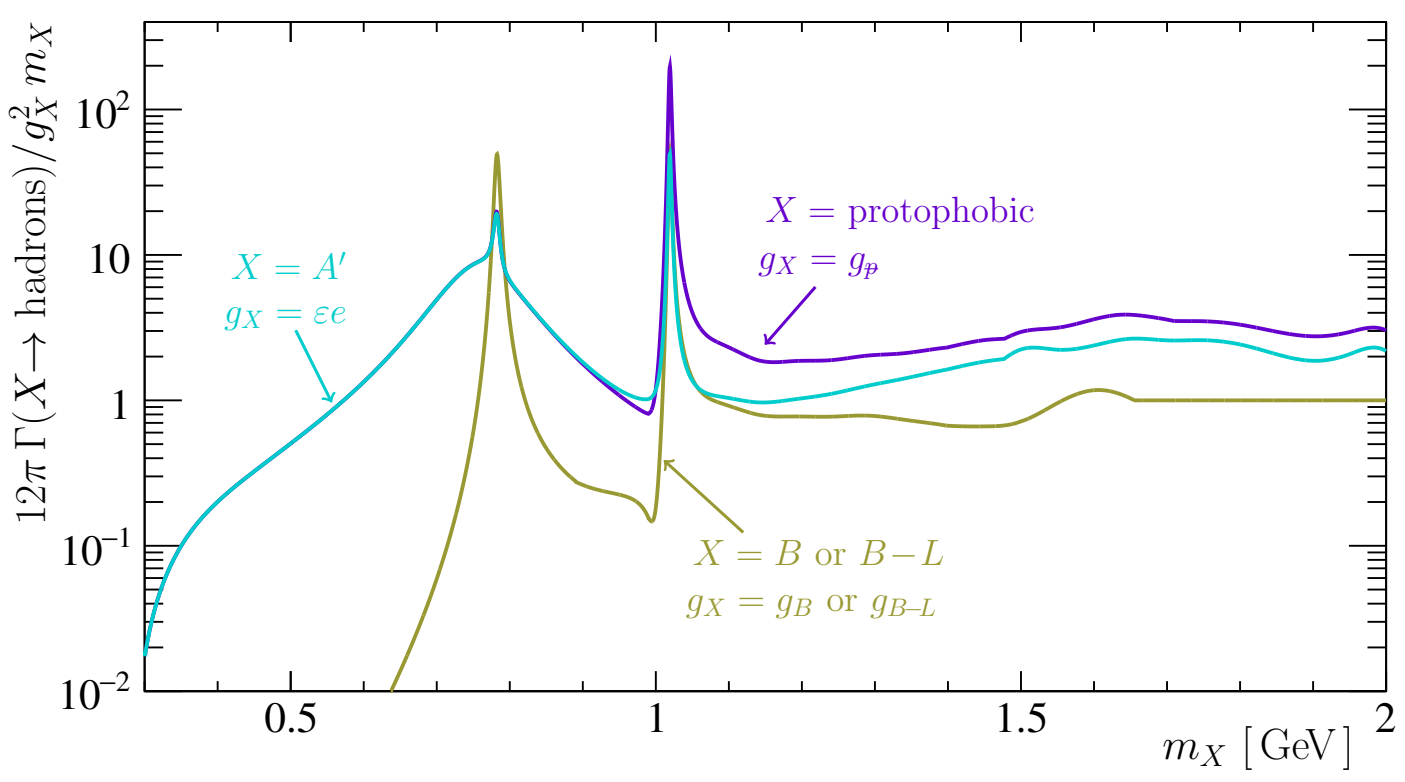

Figure 9. Hadronic decay width for: a dark photon, where $g_{X}=\varepsilon e$; a gauged $B-L$ or $B$ boson, where $g_{X}=g_{B}$ or $g_{B-L}$; and a protophobic boson, where $g_{X}=g_{p}$.

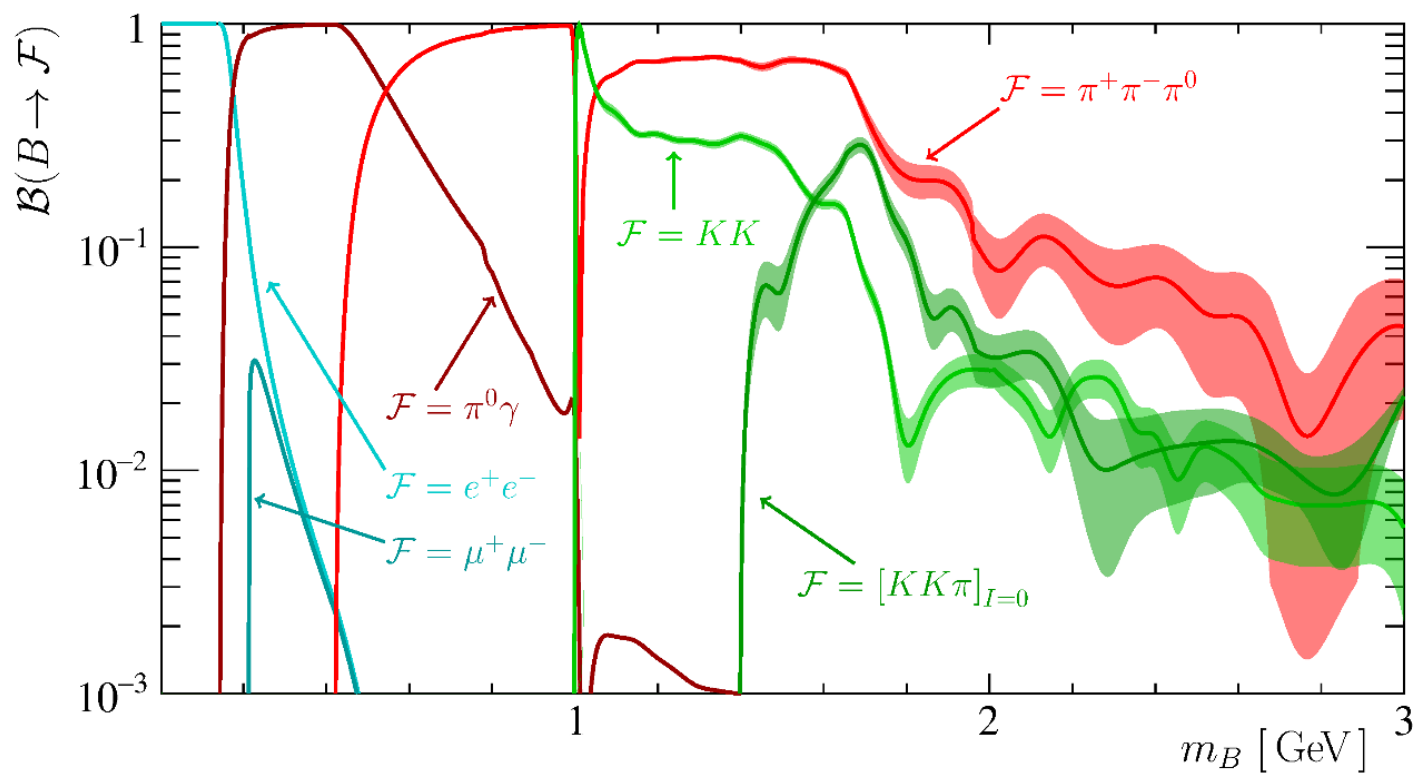

Figure 10. Decay branching fractions for the $B$ boson assuming a kinetic-mixing parameter $\varepsilon=e g_{B} /(4 \pi)^{2}$. The error bands shown for the final states $\pi^{+} \pi^{-} \pi^{0}, K K \equiv K^{+} K^{-}+K_{S} K_{L}$, and $[K K \pi]_{I=0}$ (i.e. the isoscalar component of the $K K \pi$ final state) are due to the limited experimental knowledge of the $e^{+} e^{-} \rightarrow \mathcal{F}$ cross sections. In addition, the use of VMD and U(3) symmetry introduces roughly a $20 \%$ uncertainty on all hadronic decay rates. 


\begin{tabular}{|c|c|c|c|}
\hline experiment & production & final state & efficiency ratio $\epsilon\left(\tau_{X}\right) / \epsilon\left(\tau_{A^{\prime}}\right)$ \\
\hline BaBar [29] & $e^{+} e^{-} \rightarrow X \gamma$ & $e^{+} e^{-}, \mu^{+} \mu^{-}$ & 1 \\
NA48/2 [41] & $\pi^{0} \rightarrow X \gamma$ & $e^{+} e^{-}$ & Eq. $(2.20)$ with $\tilde{t}=[1 \mathrm{~m}] /(c \gamma)$, \\
where $\gamma=50 \mathrm{GeV} / m_{X}$
\end{tabular}

Table 4. Summary of experiments that searched for prompt or invisible $A^{\prime}$ decays. See appendix C.5 for discussion on the LHCb search for long-lived $A^{\prime}$ decays.

\begin{tabular}{|c|c|c|c|}
\hline experiment & production & final state & $L_{\mathrm{dec}} / L_{\mathrm{sh}}$ \\
\hline E141 [12], E137 [13], E774 [14], & $e Z \rightarrow e Z X[9,118]$ & $e^{+} e^{-}$ & $\frac{35}{0.12}, \frac{204}{179}, \frac{2}{0.3}$, \\
KEK [11], Orsay [15] & & $\frac{2.2}{2.4}, \frac{2}{1}$ \\
NA64 [23] & $\pi^{0} \rightarrow X \gamma[20]$, & $e^{+} e^{-}$ & $\frac{23}{64}$ \\
$\nu$-CAL I [119, 120] & $p Z \rightarrow p Z X[22]$ & & $\frac{10}{480}$ \\
CHARM [121] & $\eta^{(\prime)} \rightarrow X \gamma[21]$ & $e^{+} e^{-}$ & $\frac{7.5}{835}, \frac{7}{128}$ \\
\hline
\end{tabular}

Table 5. Summary of beam dump experiments that searched for long-lived dark photons. 


\section{C.1 BaBar}

The BaBar collaboration published strong constraints on both visible [29] and invisible [46] $A^{\prime}$ decays. Their visible search used both $A^{\prime} \rightarrow e^{+} e^{-}$and $A^{\prime} \rightarrow \mu^{+} \mu^{-}$, and required that the leptons were consistent with originating from the beam interaction region. Even for models where $\Gamma_{X \rightarrow \text { hadrons }}=0$, the BaBar visible search is not sensitive to $X$ bosons with lifetimes large enough to qualify as non-prompt; therefore, the efficiency ratio $\epsilon\left(\tau_{X}\right) / \epsilon\left(\tau_{A^{\prime}}\right)$ is unity. The BaBar visible results combine the two $\ell^{+} \ell^{-}$final states. To recast this search for the case where $x_{e} \neq x_{\mu}$, the individual limits provided in the Supplemental Material of ref. [29] should be used. In this work, we only consider models with $x_{e}=x_{\mu}$, where the recasted constraints are obtained by solving

$$
\left(g_{X} x_{e}\right)^{2}=(\varepsilon e)^{2} \frac{\mathcal{B}\left(A^{\prime} \rightarrow \ell^{+} \ell^{-}\right)}{\mathcal{B}\left(X \rightarrow \ell^{+} \ell^{-}\right)} .
$$

For the invisible search, the assumption is again that the efficiency ratio is unity and the branching-fraction ratio above is replaced by the equivalent ratio into invisible final states.

\section{C.2 NA48/2}

The NA48/2 experiment searched for $\pi^{0} \rightarrow A^{\prime} \gamma$ followed by prompt $A^{\prime} \rightarrow e^{+} e^{-}$decays [41]. The prompt requirement maintains high efficiency until the flight distance reaches about $1 \mathrm{~m}$. The maximum $\gamma$ factors are about $50 \mathrm{GeV} / m_{X}$. We take the prompt-criteria efficiency to be given by eq. (2.20) with $\tilde{t}=[1 \mathrm{~m}] /(c \gamma)$ and $\gamma=50 \mathrm{GeV} / m_{X}$, which is unity for the $A^{\prime}$. This efficiency factor, however, is important for a leptophobic boson, since the production utilizes the quark couplings whereas the decay must go to $e^{+} e^{-}$, which is suppressed as it arises due to kinetic mixing. Recasting these limits for an $X$ boson is done using eq. (2.10), see also table 3 , with the appropriate mass- and model-dependent values of $\mathcal{B}\left(X \rightarrow e^{+} e^{-}\right)$ and $\mathcal{B}\left(A^{\prime} \rightarrow e^{+} e^{-}\right)$.

\section{C.3 Electron bremsstrahlung}

The A1 [25] and APEX [24] experiments provide the best electron bremsstrahlung constraints on promptly decaying dark photons. The decay $A^{\prime} \rightarrow e^{+} e^{-}$was searched for by both experiments, and the recasting is done using

$$
\left(g_{X} x_{e}\right)^{2}=(\varepsilon e)^{2} \frac{\mathcal{B}\left(A^{\prime} \rightarrow \ell^{+} \ell^{-}\right)}{\mathcal{B}\left(X \rightarrow \ell^{+} \ell^{-}\right)} .
$$

Neither experiment provides detailed information about prompt-like requirements; however, since the same coupling is used to produce and decay the boson, it is safe to simply take the efficiency ratio with the $A^{\prime}$ to be unity for all $X$ models. Additionally, the NA64 experiment at CERN used $100 \mathrm{GeV}$ electrons incident on an active target to search for invisible $A^{\prime}$ decays $[45,110]$. For this search, the assumption is that the efficiency ratio is unity and the branching-fraction ratio above is replaced by the equivalent ratio into invisible final states. 


\section{C.4 KLOE}

The KLOE experiment searched for $\phi \rightarrow A^{\prime} \eta$ followed by a prompt $A^{\prime} \rightarrow e^{+} e^{-}$decay [35]. Our interpretation of the prompt criteria is that good efficiency should be maintained provided that the flight distance is $\lesssim 8 \mathrm{~cm}$. The $\gamma$ factors here are $\left(m_{\phi}^{2}+m^{2}-\right.$ $\left.m_{\eta}^{2}\right) /\left(2 m_{\phi} m\right)$, which are $\mathcal{O}(1-10)$ in the mass range where KLOE has good sensitivity. We take the prompt-criteria efficiency to be given by eq. (2.20) with $\tilde{t}=[8 \mathrm{~cm}] /(c \gamma)$, which is unity for the $A^{\prime}$. Recasting these limits uses eq. (2.9) but taking the sum $\mathcal{B}\left(A^{\prime} \rightarrow e^{+} e^{-}\right)+\mathcal{B}\left(A^{\prime} \rightarrow \mu^{+} \mu^{-}\right)=1$, which was assumed by KLOE, along with the $\tau$ dependent efficiency factor for the $X$. N.b., since this search involves an $X$ produced via quark couplings and decaying via leptonic couplings, the $\tau$-dependent efficiency factor can be important despite being $\approx 100 \%$ efficient for the $A^{\prime}$.

KLOE also searched for $e^{+} e^{-} \rightarrow A^{\prime} \gamma$ using the $A^{\prime} \rightarrow \pi^{+} \pi^{-}$decay [42]. The pions were required to have their points of closest approach to the beam line within a cylindrical volume of radius $8 \mathrm{~cm}$ and length $15 \mathrm{~cm}$. The $\gamma$ factors in this search are $\left(m_{\phi}^{2}+m^{2}\right) /\left(2 m_{\phi} m\right) \lesssim 1.2$, which means that inefficiency due to the prompt criteria should only arise for $c \tau_{X} \gtrsim \mathcal{O}(10 \mathrm{~cm})$, which is not the case for any of the models studied in this work. Recasting these results is done using

$$
\left(g_{X} x_{e}\right)^{2}=(\varepsilon e)^{2} \frac{\mathcal{B}\left(A^{\prime} \rightarrow \pi^{+} \pi^{-}\right)}{\mathcal{B}\left(X \rightarrow \pi^{+} \pi^{-}\right)} .
$$

This search is useful because it fills in the gap near the $\omega$ peak in the $A^{\prime}$ constraints. Finally, KLOE performed a similar search looking for prompt $A^{\prime} \rightarrow e^{+} e^{-}$decay [32]. In this search, the cylindrical decay volume used had a radius of $1 \mathrm{~cm}$ and a length of $12 \mathrm{~cm}$, which is sufficiently large that it does not induce any lifetime-based inefficiencies in any of the models studied here.

\section{C.5 LHCb}

An inclusive search for dark photons using the $A^{\prime} \rightarrow \mu^{+} \mu^{-}$decay was performed by the LHCb experiment [31]. Both prompt and long-lived limits were published, where the latter provide $r_{\mathrm{ex}}^{\mathrm{ul}}$ as a function of $m_{A^{\prime}}$ and $\varepsilon^{2}$. Consequently, the only information needed to recast the $\mathrm{LHCb}$ results is the relative fraction of each $A^{\prime}$ production mechanism as a function of $m_{A^{\prime}}$, as given in figure 11. We determine these ratios by fitting the inclusive $\mu^{+} \mu^{-}$background-subtracted mass spectrum published by LHCb, using Monte Carlo signal templates generated using Pутніа 8 [122]. Only templates for the following predominant production mechanisms are considered in the fit: $\eta \rightarrow \mu^{+} \mu^{-} \gamma, \eta \rightarrow \mu^{+} \mu^{-}, \omega \rightarrow \mu^{+} \mu^{-} \pi^{0}$, $\omega \rightarrow \mu^{+} \mu^{-}, \rho \rightarrow \mu^{+} \mu^{-}, \phi \rightarrow \mu^{+} \mu^{-}$and Drell-Yan.

All of the fiducial requirements applied in the LHCb analysis are applied to the Monte Carlo dimuons when obtaining the templates. The nominal fractions are obtained using the cross-sections predicted with PyтнIA 8, combined with the relevant measured branching fractions [78]. Each template is smeared to account for the LHCb mass resolution. The $\eta \rightarrow \mu^{+} \mu^{-} \gamma$ and $\omega \rightarrow \mu^{+} \mu^{-} \pi^{0}$ mass shapes from PyтніA 8 are generated using a generic VMD-based Dalitz decay, and so these two templates are corrected using the mass shapes 
obtained from an NA60 analysis [123]. Similarly, the $\rho \rightarrow \mu^{+} \mu^{-}$mass shape is also corrected using the same NA60 analysis, while the $\omega \rightarrow \mu^{+} \mu^{-}$and $\phi \rightarrow \mu^{+} \mu^{-}$mass shapes are corrected using the results of ref. [85].

The LHCb mass spectrum is fitted by allowing the fraction for each template to vary within 0 to 10 times its nominal value, where the total Drell-Yan production is considered as a single template. The same isolation criterion applied in the LHCb analysis above the $\phi$ mass was also applied to the Monte Carlo dimuons when building the templates. However, the isolation quantity is not expected to match exactly between the Monte Carlo and data due to reconstruction effects. Therefore, the efficiency of this isolation requirement is also allowed to vary in the fit, resulting in a total of 8 free parameters. A validation of the fit is that the ratios of the two $\eta$ and the two $\phi$ channels match their respective known values within uncertainties.

From the Supplemental Material to ref. [31], one can see that the LHCb promptselectrion criteria are the same as those we proposed in ref. [66]; therefore, we use our simulation samples from that study and find that the efficiency is well approximated by eq. (2.20) with $\tilde{t} \approx\left\{[4 \mathrm{MeV}] /\left(m_{X}-2 m_{\mu}\right)+0.1\right\}$ ps. As discussed above, since LHCb published $r_{\mathrm{ex}}^{\mathrm{ul}}$ as a function of $m_{A^{\prime}}$ and $\varepsilon^{2}$, recasting the long-lived $A^{\prime}$ search can be done using eq. (2.21). For $\tau_{X}$ values that fall outside of the range where LHCb provided results, the efficiency ratios are taken to be

$$
\frac{\epsilon\left(\tau_{X}\right)}{\epsilon\left(\tau_{A^{\prime}}^{\min , \max }\right)} \approx \begin{cases}e^{1-\left(\tau_{A^{\prime}}^{\min } / \tau_{X}\right)} & \text { for } \tau_{X}<\tau_{A^{\prime}}^{\min }, \\ \frac{1-e^{-\tau_{A^{\prime}}^{\max } / \tau_{X}}}{1-e^{-1}} & \text { for } \tau_{X}>\tau_{A^{\prime}}^{\max },\end{cases}
$$

which correspond to a long-lived selection efficiency of zero for decay times less than the minimum reported by $\mathrm{LHCb}$ (justified by the efficiency figure provided in the Supplemental Material of ref. [31]) and to a maximum decay time that results in the muons being reconstructed by the first LHCb tracking system being less than the maximum $\tau_{A^{\prime}}$ reported by LHCb (confirmed to be a good approximation by our simulation from ref. [66]).

\section{C.6 Beam dumps}

Approximate limits are set for beam-dump experiments using eq. (2.24), where the efficiencies are determined using eq. (2.23).

\section{C.6.1 Electron beam dumps}

Limits on dark photons have been set in refs. [9, 118] using data from the E141, E137, E774, KEK, and Orsay electron beam-dump experiments [11-15]. Recasting these for an $X$ boson requires solving

$$
\left(g_{X} x_{e}\right)^{2} \mathcal{B}\left(X \rightarrow e^{+} e^{-}\right) \epsilon\left[\tau_{X}\left(g_{X}\right)\right] \geq\left(\varepsilon_{\max } e\right)^{2} \mathcal{B}\left(A^{\prime} \rightarrow e^{+} e^{-}\right) \epsilon\left[\tau_{A^{\prime}}\left(\varepsilon_{\max }\right)\right],
$$

at each mass. N.b., all of these experiments were only sensitive to decays into electrons and photons. In addition, recently the NA64 collaboration published long-lived $A^{\prime}$ constraints using $A^{\prime} \rightarrow e^{+} e^{-}$[23]. The length of the shielding (provided by a calorimeter) changed during the run, but on average it was about $0.25 \mathrm{~m}$. The total decay volume (before the 


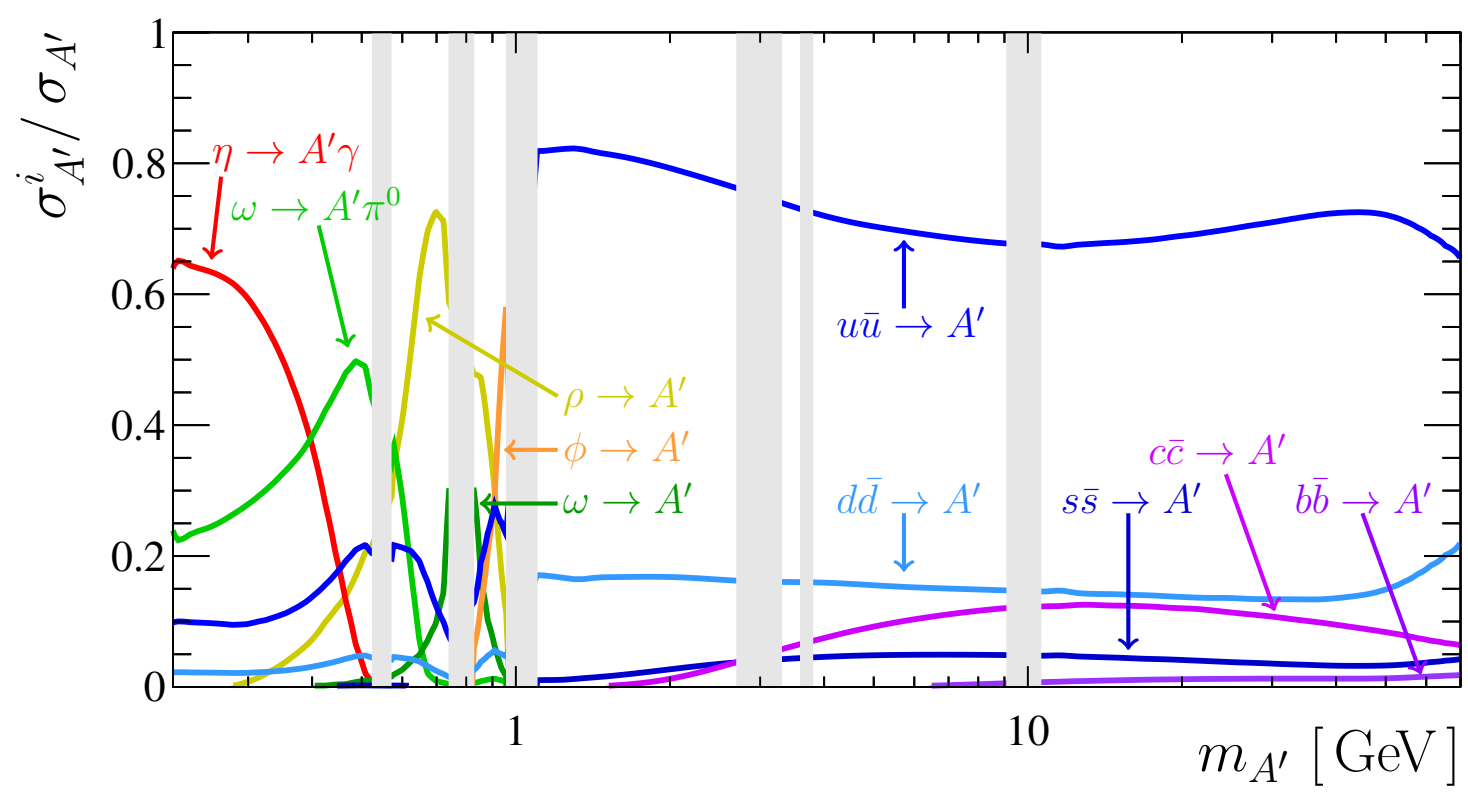

Figure 11. Dark-photon production fractions for the most important processes at LHCb in the fiducial region of ref. [31].

electromagnetic calorimeter) was $3.5 \mathrm{~m}$; however, to satisfy the selection criteria, the decay needed to happen prior to the first tracking station, which was about $1 \mathrm{~m}$ from the shielding during this run.

\section{C.6.2 Proton beam dumps}

Limits on $A^{\prime} \rightarrow e^{+} e^{-}$decays have been set by the following experiments: $\nu$-CAL I [119, 120], using $\pi^{0} \rightarrow A^{\prime} \gamma$ decays [20] and proton bremsstrahlung [22]; CHARM [121], using $\eta^{(\prime)} \rightarrow A^{\prime} \gamma$ decays [21]; and NOMAD [17] and PS191 [33] using $\pi^{0} \rightarrow A^{\prime} \gamma$ decays [36]. Recasting these for an $X$ boson involves solving

$$
\Gamma_{P \rightarrow X \gamma}\left(g_{X}\right) \mathcal{B}\left(X \rightarrow e^{+} e^{-}\right) \epsilon\left[\tau_{X}\left(g_{X}\right)\right] \geq \Gamma_{P \rightarrow A^{\prime} \gamma}\left(\varepsilon_{\max }\right) \mathcal{B}\left(A^{\prime} \rightarrow e^{+} e^{-}\right) \epsilon\left[\tau_{A^{\prime}}\left(\varepsilon_{\max }\right)\right],
$$

where $P=\pi^{0}, \eta$, or $\eta^{\prime}$ for meson-decay production, and

$$
g_{X}^{2}\left(2 x_{u}+x_{d}\right)^{2} \mathcal{B}\left(X \rightarrow e^{+} e^{-}\right) \epsilon\left[\tau_{X}\left(g_{X}\right)\right] \geq\left(\varepsilon_{\max } e\right)^{2} \mathcal{B}\left(A^{\prime} \rightarrow e^{+} e^{-}\right) \epsilon\left[\tau_{A^{\prime}}\left(\varepsilon_{\max }\right)\right],
$$

for proton bremsstrahlung.

\section{C.7 LEP}

Mono-photon searches from LEP $[116,117]$ were used to set limits on dark photons that decay invisibly in ref. [52]. Here, we assume on-shell $A^{\prime}$ production, and rescale the results of ref. [52] assuming $g_{\chi} \gg g_{e}$ and $m_{\chi} \ll m_{A^{\prime}}$. Since ref. [52] only reports results for $m_{X}=10,50$ and $100 \mathrm{GeV}$, we simply interpolate to obtain results for other masses.

Open Access. This article is distributed under the terms of the Creative Commons Attribution License (CC-BY 4.0), which permits any use, distribution and reproduction in any medium, provided the original author(s) and source are credited. 


\section{References}

[1] R. Essig et al., Working group report: new light weakly coupled particles, in the proceedings of the 2013 Community Summer Study on the Future of U.S. Particle Physics: Snowmass on the Mississippi (CSS2013), July 29-August 6, Minneapolis, U.S.A. (2013), arXiv: 1311.0029 [INSPIRE].

[2] J. Alexander et al., Dark Sectors 2016 workshop: community report, 2016, arXiv: 1608.08632, http://inspirehep.net/record/1484628/files/arXiv:1608.08632.pdf [INSPIRE].

[3] M. Battaglieri et al., US cosmic visions: new ideas in dark matter 201\%: community report, arXiv: 1707.04591 [INSPIRE].

[4] L.B. Okun, Limits of electrodynamics: paraphotons?, Sov. Phys. JETP 56 (1982) 502 [INSPIRE].

[5] P. Galison and A. Manohar, Two Z's or not two Z's?, Phys. Lett. 136B (1984) 279 [INSPIRE].

[6] B. Holdom, Two U(1)'s and epsilon charge shifts, Phys. Lett. 166B (1986) 196 [INSPIRE].

[7] M. Pospelov, A. Ritz and M.B. Voloshin, Secluded WIMP dark matter, Phys. Lett. B 662 (2008) 53 [arXiv:0711.4866] [InSPIRE].

[8] N. Arkani-Hamed, D.P. Finkbeiner, T.R. Slatyer and N. Weiner, A theory of dark matter, Phys. Rev. D 79 (2009) 015014 [arXiv:0810.0713] [INSPIRE].

[9] J.D. Bjorken, R. Essig, P. Schuster and N. Toro, New fixed-target experiments to search for dark gauge forces, Phys. Rev. D 80 (2009) 075018 [arXiv:0906.0580] [InSPIRE].

[10] CHARM collaboration, F. Bergsma et al., A search for decays of heavy neutrinos in the mass range $0.5 \mathrm{GeV}$ to $2.8 \mathrm{GeV}$, Phys. Lett. 166B (1986) 473 [INSPIRE].

[11] A. Konaka et al., Search for neutral particles in electron beam dump experiment, Phys. Rev. Lett. 57 (1986) 659 [INSPIRE].

[12] E.M. Riordan et al., A search for short lived axions in an electron beam dump experiment, Phys. Rev. Lett. 59 (1987) 755 [INSPIRE].

[13] J.D. Bjorken et al., Search for neutral metastable penetrating particles produced in the SLAC beam dump, Phys. Rev. D 38 (1988) 3375 [InSPIRE].

[14] A. Bross et al., A search for shortlived particles produced in an electron beam dump, Phys. Rev. Lett. 67 (1991) 2942 [INSPIRE].

[15] M. Davier and H. Nguyen Ngoc, An unambiguous search for a light Higgs boson, Phys. Lett. B 229 (1989) 150 [INSPIRE].

[16] LSND collaboration, C. Athanassopoulos et al., Evidence for muon-neutrino $\rightarrow$ electron-neutrino oscillations from pion decay in flight neutrinos, Phys. Rev. C 58 (1998) 2489 [nucl-ex/9706006] [INSPIRE].

[17] NOMAD collaboration, P. Astier et al., Search for heavy neutrinos mixing with tau neutrinos, Phys. Lett. B 506 (2001) 27 [hep-ex/0101041] [INSPIRE].

[18] R. Essig, R. Harnik, J. Kaplan and N. Toro, Discovering new light states at neutrino experiments, Phys. Rev. D 82 (2010) 113008 [arXiv: 1008.0636] [INSPIRE]. 
[19] M. Williams, C.P. Burgess, A. Maharana and F. Quevedo, New constraints (and motivations) for abelian gauge bosons in the MeV-TeV mass range, JHEP 08 (2011) 106 [arXiv:1103.4556] [INSPIRE].

[20] J. Blümlein and J. Brunner, New exclusion limits for dark gauge forces from beam-dump data, Phys. Lett. B 701 (2011) 155 [arXiv:1104.2747] [inSPIRE].

[21] S.N. Gninenko, Constraints on sub-GeV hidden sector gauge bosons from a search for heavy neutrino decays, Phys. Lett. B 713 (2012) 244 [arXiv:1204.3583] [InSPIRE].

[22] J. Blümlein and J. Brunner, New exclusion limits on dark gauge forces from proton bremsstrahlung in beam-dump data, Phys. Lett. B 731 (2014) 320 [arXiv:1311.3870] [INSPIRE].

[23] NA64 collaboration, D. Banerjee et al., Search for a new X(16.7) boson and dark photons in the NA64 experiment at CERN, arXiv: 1803.07748 [INSPIRE].

[24] APEX collaboration, S. Abrahamyan et al., Search for a new gauge boson in electron-nucleus fixed-target scattering by the APEX experiment, Phys. Rev. Lett. 107 (2011) 191804 [arXiv:1108.2750] [INSPIRE].

[25] H. Merkel et al., Search at the Mainz Microtron for light massive gauge bosons relevant for the muon g-2 anomaly, Phys. Rev. Lett. 112 (2014) 221802 [arXiv:1404.5502] [INSPIRE].

[26] A1 collaboration, H. Merkel et al., Search for light gauge bosons of the dark sector at the Mainz Microtron, Phys. Rev. Lett. 106 (2011) 251802 [arXiv:1101.4091] [INSPIRE].

[27] BABAR collaboration, B. Aubert et al., Search for dimuon decays of a light scalar boson in radiative transitions $\Upsilon \rightarrow \gamma A^{0}$, Phys. Rev. Lett. 103 (2009) 081803 [arXiv:0905.4539] [INSPIRE].

[28] D. Curtin et al., Exotic decays of the 125 GeV Higgs boson, Phys. Rev. D 90 (2014) 075004 [arXiv: 1312.4992] [INSPIRE].

[29] BABAR collaboration, J.P. Lees et al., Search for a dark photon in $e^{+} e^{-}$collisions at BaBar, Phys. Rev. Lett. 113 (2014) 201801 [arXiv:1406.2980] [InSPIRE].

[30] BESIII collaboration, M. Ablikim et al., Dark photon search in the mass range between 1.5 and $3.4 \mathrm{GeV} / \mathrm{c}^{2}$, Phys. Lett. B 774 (2017) 252 [arXiv: 1705.04265] [INSPIRE].

[31] LHCb collaboration, Search for dark photons produced in $13 \mathrm{TeV}$ pp collisions, Phys. Rev. Lett. 120 (2018) 061801 [arXiv:1710.02867] [INSPIRE].

[32] A. Anastasi et al., Limit on the production of a low-mass vector boson in $e^{+} e^{-} \rightarrow U \gamma$, $U \rightarrow e^{+} e^{-}$with the KLOE experiment, Phys. Lett. B 750 (2015) 633 [arXiv:1509.00740] [INSPIRE].

[33] G. Bernardi et al., Search for neutrino decay, Phys. Lett. 166B (1986) 479 [INSPIRE].

[34] SINDRUM I collaboration, R. Meijer Drees et al., Search for weakly interacting neutral bosons produced in $\pi^{-} p$ interactions at rest and decaying into $e^{+} e^{-}$pairs., Phys. Rev. Lett. 68 (1992) 3845 [INSPIRE].

[35] KLOE-2 collaboration, F. Archilli et al., Search for a vector gauge boson in $\phi$ meson decays with the KLOE detector, Phys. Lett. B 706 (2012) 251 [arXiv:1110.0411] [INSPIRE].

[36] S.N. Gninenko, Stringent limits on the $\pi^{0} \rightarrow \gamma X, X \rightarrow e^{+} e^{-}$decay from neutrino experiments and constraints on new light gauge bosons, Phys. Rev. D 85 (2012) 055027 [arXiv:1112.5438] [INSPIRE]. 
[37] KLOE-2 collaboration, D. Babusci et al., Limit on the production of a light vector gauge boson in phi meson decays with the KLOE detector, Phys. Lett. B 720 (2013) 111 [arXiv:1210.3927] [INSPIRE].

[38] WASA-AT-COSY collaboration, P. Adlarson et al., Search for a dark photon in the $\pi^{0} \rightarrow e^{+} e^{-} \gamma$ decay, Phys. Lett. B 726 (2013) 187 [arXiv:1304.0671] [InSPIRE].

[39] HADES collaboration, G. Agakishiev et al., Searching a dark photon with HADES, Phys. Lett. B 731 (2014) 265 [arXiv:1311.0216] [INSPIRE].

[40] PHENIX collaboration, A. Adare et al., Search for dark photons from neutral meson decays in $p+p$ and $d+$ Au collisions at $\sqrt{s_{N N}}=200 \mathrm{GeV}$, Phys. Rev. C 91 (2015) 031901 [arXiv: 1409.0851] [INSPIRE].

[41] NA48/2 collaboration, J.R. Batley et al., Search for the dark photon in $\pi^{0}$ decays, Phys. Lett. B 746 (2015) 178 [arXiv: 1504.00607] [INSPIRE].

[42] KLOE-2 collaboration, A. Anastasi et al., Limit on the production of a new vector boson in $e^{+} e^{-} \rightarrow U \gamma, U \rightarrow \pi^{+} \pi^{-}$with the KLOE experiment, Phys. Lett. B 757 (2016) 356 [arXiv: 1603.06086] [INSPIRE].

[43] R. Essig et al., Constraining light dark matter with low-energy $e^{+} e^{-}$colliders, JHEP 11 (2013) 167 [arXiv: 1309.5084] [inSPIRE].

[44] H. Davoudiasl, H.-S. Lee and W.J. Marciano, Muon g-2, rare kaon decays and parity violation from dark bosons, Phys. Rev. D 89 (2014) 095006 [arXiv:1402.3620] [InSPIRE].

[45] NA64 collaboration, D. Banerjee et al., Search for invisible decays of sub-GeV dark photons in missing-energy events at the CERN SPS, Phys. Rev. Lett. 118 (2017) 011802 [arXiv: 1610.02988] [INSPIRE].

[46] BABAR collaboration, J.P. Lees et al., Search for invisible decays of a dark photon produced in $e^{+} e^{-}$collisions at BaBar, Phys. Rev. Lett. 119 (2017) 131804 [arXiv:1702.03327] [INSPIRE].

[47] E787 collaboration, S. Adler et al., Further evidence for the decay $K^{+} \rightarrow \pi^{+} \nu \bar{\nu}$, Phys. Rev. Lett. 88 (2002) 041803 [hep-ex/0111091] [INSPIRE].

[48] E787 collaboration, S. Adler et al., Further search for the decay $K^{+} \rightarrow \pi^{+} \nu \bar{\nu}$ in the momentum region $P<195 \mathrm{MeV} / \mathrm{c}$, Phys. Rev. D 70 (2004) 037102 [hep-ex/0403034] [INSPIRE].

[49] BNL-E949 collaboration, A.V. Artamonov et al., Study of the decay $K^{+} \rightarrow \pi^{+} \nu \bar{\nu}$ in the momentum region $140<P_{\pi}<199 \mathrm{MeV} / \mathrm{c}$, Phys. Rev. D 79 (2009) 092004 [arXiv: 0903.0030] [INSPIRE].

[50] P. Fayet, Constraints on Light Dark Matter and U bosons, from $\psi, \Upsilon, K^{+}, \pi^{0}, \eta$ and $\eta^{\prime}$ decays, Phys. Rev. D 74 (2006) 054034 [hep-ph/0607318] [INSPIRE].

[51] P. Fayet, U-boson production in $e^{+} e^{-}$annihilations, $\psi$ and $\Upsilon$ decays and light dark matter, Phys. Rev. D 75 (2007) 115017 [hep-ph/0702176] [INSPIRE].

[52] P.J. Fox et al., LEP shines light on dark matter, Phys. Rev. D 84 (2011) 014028 [arXiv:1103.0240] [INSPIRE].

[53] R. Essig, P. Schuster, N. Toro and B. Wojtsekhowski, An electron fixed target experiment to search for a new vector boson $A^{\prime}$ decaying to $e^{+} e^{-}$, JHEP 02 (2011) 009 [arXiv: 1001.2557] [INSPIRE]. 
[54] M. Freytsis, G. Ovanesyan and J. Thaler, Dark force detection in low energy ep collisions, JHEP 01 (2010) 111 [arXiv:0909.2862] [INSPIRE].

[55] J. Balewski et al., DarkLight: a search for dark forces at the Jefferson Laboratory Free-Electron Laser Facility, arXiv:1307.4432 [INSPIRE].

[56] B. Wojtsekhowski, D. Nikolenko and I. Rachek, Searching for a new force at VEPP-3, arXiv: 1207.5089 [INSPIRE].

[57] T. Beranek, H. Merkel and M. Vanderhaeghen, Theoretical framework to analyze searches for hidden light gauge bosons in electron scattering fixed target experiments, Phys. Rev. D 88 (2013) 015032 [arXiv: 1303.2540] [INSPIRE].

[58] B. Echenard, R. Essig and Y.-M. Zhong, Projections for dark photon searches at Muße, JHEP 01 (2015) 113 [arXiv:1411.1770] [INSPIRE].

[59] M. Battaglieri et al., The heavy photon search test detector, Nucl. Instrum. Meth. A 777 (2015) 91 [arXiv: 1406.6115] [INSPIRE].

[60] S. Alekhin et al., A facility to search for hidden particles at the CERN SPS: the SHiP physics case, Rept. Prog. Phys. 79 (2016) 124201 [arXiv: 1504.04855] [INSPIRE].

[61] S. Gardner, R.J. Holt and A.S. Tadepalli, New prospects in fixed target searches for dark forces with the SeaQuest experiment at Fermilab, Phys. Rev. D 93 (2016) 115015 [arXiv: 1509.00050] [INSPIRE].

[62] P. Ilten, J. Thaler, M. Williams and W. Xue, Dark photons from charm mesons at LHCb, Phys. Rev. D 92 (2015) 115017 [arXiv:1509.06765] [InSPIRE].

[63] D. Curtin, R. Essig, S. Gori and J. Shelton, Illuminating dark photons with high-energy colliders, JHEP 02 (2015) 157 [arXiv: 1412.0018] [INSPIRE].

[64] M. He, X.-G. He and C.-K. Huang, Dark photon search at a circular $e^{+} e^{-}$collider, Int. J. Mod. Phys. A 32 (2017) 1750138 [arXiv:1701.08614] [INSPIRE].

[65] J. Kozaczuk, Dark photons from nuclear transitions, Phys. Rev. D 97 (2018) 015014 [arXiv: 1708.06349] [INSPIRE].

[66] P. Ilten, Y. Soreq, J. Thaler, M. Williams and W. Xue, Proposed inclusive dark photon search at LHCb, Phys. Rev. Lett. 116 (2016) 251803 [arXiv:1603.08926] [INSPIRE].

[67] J. Feng, I. Galon, F. Kling and S. Trojanowski, ForwArd search ExpeRiment at the LHC, Phys. Rev. D 97 (2018) 035001 [arXiv: 1708.09389] [InSPIRE].

[68] M. Raggi and V. Kozhuharov, Proposal to search for a dark photon in positron on target

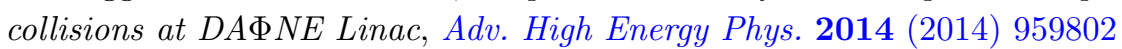
[arXiv: 1403.3041] [INSPIRE].

[69] J. Alexander, MMAPS: Missing-Mass A-Prime Search, EPJ Web Conf. 142 (2017) 01001.

[70] G. Barello, S. Chang and C.A. Newby, Correlated signals at the energy and intensity frontiers from non-Abelian kinetic mixing, Phys. Rev. D 94 (2016) 055018 [arXiv: 1511.02865] [INSPIRE].

[71] S. Cassel, D.M. Ghilencea and G.G. Ross, Electroweak and dark matter constraints on a $Z^{\prime}$ in models with a hidden valley, Nucl. Phys. B 827 (2010) 256 [arXiv:0903.1118] [InSPIRE].

[72] J.M. Cline, G. Dupuis, Z. Liu and W. Xue, The windows for kinetically mixed $Z^{\prime}$-mediated dark matter and the galactic center $\gamma$ ray excess, JHEP 08 (2014) 131 [arXiv:1405.7691] [INSPIRE]. 
[73] J.A. Dror, R. Lasenby and M. Pospelov, Dark forces coupled to nonconserved currents, Phys. Rev. D 96 (2017) 075036 [arXiv:1707.01503] [InSPIRE].

[74] J.A. Dror, R. Lasenby and M. Pospelov, New constraints on light vectors coupled to anomalous currents, Phys. Rev. Lett. 119 (2017) 141803 [arXiv:1705.06726] [INSPIRE].

[75] A. Ismail and A. Katz, Anomalous $Z^{\prime}$ and diboson resonances at the LHC, JHEP 04 (2018) 122 [arXiv: 1712.01840] [INSPIRE].

[76] S. Tulin, New weakly-coupled forces hidden in low-energy QCD, Phys. Rev. D 89 (2014) 114008 [arXiv: 1404.4370] [INSPIRE].

[77] T. Fujiwara et al., Nonabelian anomaly and vector mesons as dynamical gauge bosons of hidden local symmetries, Prog. Theor. Phys. 73 (1985) 926 [InSPIRE].

[78] Particle Data Group collaboration, C. Patrignani et al., Review of particle physics, Chin. Phys. C 40 (2016) 100001 [inSPIRE].

[79] BaBAR collaboration, J.P. Lees et al., Precise measurement of the $e^{+} e^{-} \rightarrow \pi^{+} \pi^{-}(\gamma)$ cross section with the initial-state radiation method at BABAR, Phys. Rev. D 86 (2012) 032013 [arXiv: 1205.2228] [INSPIRE].

[80] BABAr collaboration, B. Aubert et al., Study of $e^{+} e^{-} \rightarrow \pi^{+} \pi^{-} \pi^{0}$ process using initial state radiation with BaBar, Phys. Rev. D 70 (2004) 072004 [hep-ex/0408078] [INSPIRE].

[81] BABAR collaboration, J.P. Lees et al., Precision measurement of the $e^{+} e^{-} \rightarrow K^{+} K^{-}(\gamma)$ cross section with the initial-state radiation method at BABAR, Phys. Rev. D 88 (2013) 032013 [arXiv: 1306.3600] [INSPIRE].

[82] BABAR collaboration, B. Aubert et al., Measurements of $e^{+} e^{-} \rightarrow K^{+} K^{-} \eta, K^{+} K^{-} \pi^{0}$ and $K_{s}^{0} K^{ \pm} \pi^{\mp}$ cross- sections using initial state radiation events, Phys. Rev. D 77 (2008) 092002 [arXiv: 0710.4451] [INSPIRE].

[83] BABAR collaboration, J.P. Lees et al., Initial-state radiation measurement of the $e^{+} e^{-} \rightarrow \pi^{+} \pi^{-} \pi^{+} \pi^{-}$cross section, Phys. Rev. D 85 (2012) 112009 [arXiv:1201.5677] [INSPIRE].

[84] BABAR collaboration, J.P. Lees et al., Measurement of the $e^{+} e^{-} \rightarrow \pi^{+} \pi^{-} \pi^{0} \pi^{0}$ cross section using initial-state radiation at BABAR, Phys. Rev. D 96 (2017) 092009 [arXiv: 1709.01171] [INSPIRE].

[85] M.N. Achasov et al., Study of the process $e^{+} e^{-} \rightarrow \pi^{+} \pi^{-} \pi^{0}$ in the energy region $s^{1 / 2}$ below 0.98 GeV, Phys. Rev. D 68 (2003) 052006 [hep-ex/0305049] [InSPIRE].

[86] M.N. Achasov et al., Study of the process $e^{+} e^{-} \rightarrow \pi^{+} \pi^{-} \pi^{0}$ in the energy region $s^{1 / 2}$ from $0.98 \mathrm{GeV}$ to $1.38 \mathrm{GeV}$, Phys. Rev. D 66 (2002) 032001 [hep-ex/0201040] [InSPIRE].

[87] J.L. Feng et al., Particle physics models for the $17 \mathrm{MeV}$ anomaly in beryllium nuclear decays, Phys. Rev. D 95 (2017) 035017 [arXiv:1608.03591] [InSPIRE].

[88] J. Heeck, Unbroken B-L symmetry, Phys. Lett. B 739 (2014) 256 [arXiv:1408.6845] [INSPIRE].

[89] C. Fanelli and M. Williams, Photoproduction of leptophobic bosons, J. Phys. G 44 (2017) 014002 [arXiv: 1605.07161] [INSPIRE].

[90] M. Pospelov, Secluded U(1) below the weak scale, Phys. Rev. D 80 (2009) 095002 [arXiv:0811.1030] [INSPIRE]. 
[91] M. Endo, K. Hamaguchi and G. Mishima, Constraints on hidden photon models from electron g- 2 and hydrogen spectroscopy, Phys. Rev. D 86 (2012) 095029

[arXiv:1209.2558] [INSPIRE].

[92] M. Bauer, P. Foldenauer and J. Jaeckel, Hunting all the hidden photons, arXiv:1803.05466 [INSPIRE].

[93] LHCb collaboration, Search for hidden-sector bosons in $B^{0} \rightarrow K^{* 0} \mu^{+} \mu^{-}$decays, Phys. Rev. Lett. 115 (2015) 161802 [arXiv:1508.04094] [INSPIRE].

[94] LHCb collaboration, Search for long-lived scalar particles in $B^{+} \rightarrow K^{+} \chi\left(\mu^{+} \mu^{-}\right)$decays, Phys. Rev. D 95 (2017) 071101 [arXiv: 1612.07818] [InSPIRE].

[95] B.A. Dobrescu and C. Frugiuele, Hidden GeV-scale interactions of quarks, Phys. Rev. Lett. 113 (2014) 061801 [arXiv: 1404.3947] [INSPIRE].

[96] R. Harnik, J. Kopp and P.A.N. Machado, Exploring $\nu$ signals in dark matter detectors, JCAP 07 (2012) 026 [arXiv: 1202.6073] [INSPIRE].

[97] G. Bellini et al., Precision measurement of the ${ }^{7}$ Be solar neutrino interaction rate in Borexino, Phys. Rev. Lett. 107 (2011) 141302 [arXiv:1104.1816] [InSPIRE].

[98] TEXONO collaboration, M. Deniz et al., Measurement of $\bar{\nu}_{e}$-e scattering cross-section with a CsI(Tl) scintillating crystal array at the Kuo-Sheng nuclear power reactor, Phys. Rev. D 81 (2010) 072001 [arXiv:0911.1597] [INSPIRE].

[99] CHARM-II collaboration, P. Vilain et al., Measurement of differential cross-sections for muon-neutrino electron scattering, Phys. Lett. B 302 (1993) 351 [INSPIRE].

[100] C. Frugiuele, E. Fuchs, G. Perez and M. Schlaffer, Constraining new physics models with isotope shift spectroscopy, Phys. Rev. D 96 (2017) 015011 [arXiv:1602.04822] [INSPIRE].

[101] E.D. Carlson, Limits on a new U(1) coupling, Nucl. Phys. B 286 (1987) 378 [INSPIRE].

[102] A. Aranda and C.D. Carone, Limits on a light leptophobic gauge boson, Phys. Lett. B 443 (1998) 352 [hep-ph/9809522] [INSPIRE].

[103] ARGUS collaboration, H. Albrecht et al., An upper limit for two jet production in Direct $\Upsilon(1 s)$ decays, Z. Phys. C 31 (1986) 181 [INSPIRE].

[104] S. Prakhov et al., Measurement of the invariant-mass spectrum for the two photons from the $\eta \rightarrow \pi^{0} \gamma \gamma$ decay, Phys. Rev. C 78 (2008) 015206 [inSPIRE].

[105] BeLle collaboration, J. Grygier et al., Search for $B \rightarrow h \nu \bar{\nu}$ decays with semileptonic tagging at Belle, Phys. Rev. D 96 (2017) 091101 [arXiv:1702.03224] [INSPIRE].

[106] KTEV collaboration, A. Alavi-Harati et al., Search for the rare decay $K(L) \rightarrow \pi^{0} e^{+} e^{-}$, Phys. Rev. Lett. 93 (2004) 021805 [hep-ex/0309072] [INSPIRE].

[107] E949 collaboration, A.V. Artamonov et al., New measurement of the $K^{+} \rightarrow \pi^{+} \nu \bar{\nu}$ branching ratio, Phys. Rev. Lett. 101 (2008) 191802 [arXiv:0808.2459] [INSPIRE].

[108] L3 collaboration, M. Acciarri et al., Search for new physics in energetic single photon production in $e^{+} e^{-}$annihilation at the $Z$ resonance, Phys. Lett. B 412 (1997) 201 [INSPIRE].

[109] DELPHI collaboration, P. Abreu et al., Search for neutral heavy leptons produced in Z decays, Z. Phys. C 74 (1997) 57 [Erratum ibid. C 75 (1997) 580] [InSPIRE]. 
[110] NA64 collaboration, D. Banerjee et al., Search for vector mediator of Dark Matter production in invisible decay mode, Phys. Rev. D 97 (2018) 072002 [arXiv:1710.00971] [INSPIRE].

[111] P. Fayet, Extra U(1)'s and new forces, Nucl. Phys. B 347 (1990) 743 [InSPIRE].

[112] M.D. Campos et al., Neutrino masses and absence of flavor changing interactions in the 2HDM from gauge principles, JHEP 08 (2017) 092 [arXiv: 1705.05388] [INSPIRE].

[113] Y. Kahn, G. Krnjaic, S. Mishra-Sharma and T.M.P. Tait, Light weakly coupled axial forces: models, constraints and projections, JHEP 05 (2017) 002 [arXiv: 1609.09072] [INSPIRE].

[114] U. Haisch and J.F. Kamenik, Searching for new spin-0 resonances at LHCb, Phys. Rev. D 93 (2016) 055047 [arXiv: 1601.05110] [INSPIRE].

[115] T. Feldmann, Quark structure of pseudoscalar mesons, Int. J. Mod. Phys. A 15 (2000) 159 [hep-ph/9907491] [INSPIRE].

[116] DELPHI collaboration, J. Abdallah et al., Photon events with missing energy in $e^{+} e^{-}$ collisions at $\sqrt{s}=130 \mathrm{GeV}$ to $209 \mathrm{GeV}$, Eur. Phys. J. C 38 (2005) 395 [hep-ex/0406019] [INSPIRE].

[117] DELPHI collaboration, J. Abdallah et al., Search for one large extra dimension with the DELPHI detector at LEP, Eur. Phys. J. C 60 (2009) 17 [arXiv:0901.4486] [InSPIRE].

[118] S. Andreas, C. Niebuhr and A. Ringwald, New limits on hidden photons from past electron beam dumps, Phys. Rev. D 86 (2012) 095019 [arXiv: 1209.6083] [InSPIRE].

[119] J. Blumlein et al., Limits on neutral light scalar and pseudoscalar particles in a proton beam dump experiment, Z. Phys. C 51 (1991) 341 [inSPIRE].

[120] J. Blumlein et al., Limits on the mass of light (pseudo)scalar particles from Bethe-Heitler $e^{+} e^{-}$and $\mu^{+} \mu^{-}$pair production in a proton-iron beam dump experiment, Int. J. Mod. Phys. A 7 (1992) 3835 [INSPIRE].

[121] CHARM collaboration, F. Bergsma et al., Search for axion like particle production in $400 \mathrm{GeV}$ proton-copper interactions, Phys. Lett. B 157 (1985) 458.

[122] T. Sjöstrand et al., An introduction to PYTHIA 8.2, Comput. Phys. Commun. 191 (2015) 159 [arXiv: 1410.3012] [INSPIRE].

[123] NA60 collaboration, R. Arnaldi et al., Precision study of the $\eta \rightarrow \mu^{+} \mu^{-} \gamma$ and $\omega \rightarrow \mu^{+} \mu^{-} \pi^{0}$ electromagnetic transition form-factors and of the $\rho \rightarrow \mu^{+} \mu^{-}$line shape in NA60, Phys. Lett. B 757 (2016) 437 [arXiv: 1608.07898] [INSPIRE]. 